\title{
Gromov-Witten invariants of blow-ups along submanifolds with convex normal bundles
}

\author{
HSIN-HONG LAI
}

\begin{abstract}
When the normal bundle $N_{Z / X}$ is convex with a minor assumption, we prove that genus $-0 \mathrm{GW}$-invariants of the blow-up $\mathrm{Bl}_{Z} X$ of $X$ along a submanifold $Z$, with cohomology insertions from $X$, are identical to GW-invariants of $X$. Under the same hypothesis, a vanishing theorem is also proved. An example to which these two theorems apply is when $N_{Z / X}$ is generated by its global sections. These two main theorems do not hold for arbitrary blow-ups, and counterexamples are included.
\end{abstract}

$14 \mathrm{~N} 35 ; 53 \mathrm{D} 45,14 \mathrm{E} 05$

\section{Introduction}

In [28], Y Ruan proposes naturality problems of quantum cohomology rings under birational surgery. GW-invariants are also used to classify symplectic manifolds in a symplectic birational geometric program in the work of $\mathrm{Hu}-\mathrm{Li}-\mathrm{Ruan}$ [14] and McDuff [27]. Recently, there has also been substantial progress in the crepant resolution conjecture, which roughly says the quantum cohomology is preserved by the crepant resolution after analytic continuation and some changes of parameters. On the other hand, the blow-up formula for GW-invariants is known only for very few cases. Let $\pi: \tilde{X} \rightarrow X$ be the blow up of $X$ along the submanifold $Z$. A natural question is if the induced genus $-0 \mathrm{GW}$-invariants of $\tilde{X}$ coincide with the GW-invariants of $X$. That is, if $\alpha_{i} \in H^{*}(X)$ and $\beta \in H_{2}(X)$, do we have

$$
\left\langle\pi^{*} \alpha_{1}, \cdots, \pi^{*} \alpha_{n}\right\rangle_{0, n, \pi ! \beta}^{\tilde{X}}=\left\langle\alpha_{1}, \cdots, \alpha_{n}\right\rangle_{0, n, \beta}^{X} ?
$$

When formulated in this generality, the answer is negative (see Bryan-Karp [3, Remark 9] or Example 4.16). In the work of Gathmann [9] and $\mathrm{Hu}$ [12; 13], the answer to Question (1) has been shown to be true in some cases, where $\operatorname{dim} Z \leq 2$ with various assumptions, including the requirement that cohomology insertions are supported away from $Z$ when $\operatorname{dim} Z=2$.

In this paper, we will show that if the normal bundle $N_{Z / X}$ is convex with a minor assumption, then the answer to Question (1) is also affirmative. This provides examples 
where $\operatorname{dim} Z$ can be any number without assuming cohomology insertions are supported away from $Z$. First recall the definition of a convex bundle:

Definition 1.1 A vector bundle $W$ over a manifold $Z$ is called convex if and only if $H^{1}\left(\mathbb{P}^{1}, f^{*} W\right)=0$ for any holomorphic map $f: \mathbb{P}^{1} \rightarrow Z$.

A vector bundle which is generated by global sections is automatically convex. Recall that a vector bundle is generated by global sections if and only if the vector bundle is the quotient sheaf of a trivial bundle. For example, the vector bundle $\bigoplus \mathcal{O}\left(a_{i}\right)$ for nonnegative $a_{i}$ is generated by global sections. In this paper, we consider two classes of submanifolds $Z \subset X$.

Definition 1.2 A connected submanifold $Z \subset X$ is of type $\mathrm{I}$, if the following two conditions are satisfied:

(1) $N_{Z / X}$ is a convex bundle over Z.

(2) There is a subbundle $\mathcal{F}$ in $N_{Z / X}$ with $\operatorname{rank} \operatorname{rk}(\mathcal{F}) \geq 2$, and $\mathcal{F}$ is generated by global sections.

An example of type $\mathrm{I}$ is when $N_{Z / X}$ is generated by global sections.

Definition 1.3 A connected submanifold $Z \subset X$ is of type II, if every holomorphic map $f: \mathbb{P}^{1} \rightarrow Z$ must be a constant map.

For example, $Z$ is of type II if $Z$ is a product of higher genus curves or abelian varieties. Our first main result is the following:

Theorem 1.4 Suppose each connected component of the submanifold $Z=\bigsqcup_{i} Z_{i} \subset X$ is of type $I$ or type II. Let $V$ be a vector bundle over $X$, and $\mathbf{c}$ be an invertible multiplicative characteristic class. Then we have an equality of genus-0 twisted Gromov-Witten invariants

$$
\left\langle\alpha_{1}, \cdots, \alpha_{n}\right\rangle_{0, n, \beta}^{X, \mathbf{c}, V}=\left\langle\pi^{*} \alpha_{1}, \cdots, \pi^{*} \alpha_{n}\right\rangle_{0, n, \pi^{!} \beta}^{\tilde{X}, \mathbf{c}, \pi^{*} V}, \text { where } \alpha_{i} \in H^{*}(X) \text { for all } i .
$$

Given an arbitrary projective manifold $X$, Example 4.12 provides several ways to find a submanifold $Z \subset X$, so that $N_{Z / X}$ is generated by global sections. This is the major source of examples to which Theorem 1.4 applies. Type I and type II cases cover most cases when $N_{Z / X}$ is convex. We speculate that Theorem 1.4 holds as long as $N_{Z / X}$ is convex without any additional assumptions. Convexity of the normal bundle is a critical assumption in Theorem 1.4. This is illustrated by Example 4.16, which has the following properties: 
(1) The submanifold $Z \subset X$ has enough freedom to move inside $X$, so that $Z$ can avoid any finite collection of holomorphic curves.

(2) The moduli spaces of $\tilde{X}$ and $X$ are both smooth and birational to each other.

(3) The difference of (pushdown) virtual classes has nonzero contribution to GWinvariants. Therefore the conclusion of Theorem 1.4 does not hold in this case.

In this example, the nonconvex part of the normal bundle $N_{Z / X}$ "twists" the obstruction bundle on the moduli space of $\tilde{X}$, and gives rise to the correction term of (pushdown) virtual classes/GW-invariants. Theorem 1.4 is a direct consequence of the following equality of virtual classes. In the following theorems, $\tilde{W}_{0}$ and $W_{0}$ are degenerations (from deformation to the normal cones) of $\tilde{X}$ and $X$ respectively. The maps $\phi$ and $\varphi$ on the corresponding moduli spaces are induced from $\pi: \tilde{X} \rightarrow X$.

Theorem 1.5 Suppose each connected component of the submanifold $Z=\bigsqcup_{i} Z_{i} \subset X$ is of type $I$ or type II. Then we have $\phi_{*}\left[\overline{\mathcal{M}}\left(\tilde{\mathcal{W}}_{0}, 0, n, \pi^{!} \beta\right)\right]^{\mathrm{vir}}=\left[\overline{\mathcal{M}}\left(\mathcal{W}_{0}, 0, n, \beta\right)\right]^{\mathrm{vir}}$.

In some special cases, Theorem 1.5 can be improved as follows:

Theorem 1.6 Suppose $Z$ is the transversal intersection of two arbitrary manifolds $X$ and $Y$ in a compact homogeneous space $\mathcal{P}$. Then we have $\varphi_{*}\left[\overline{\mathcal{M}_{0, n}}\left(\tilde{X}, \pi^{!} \beta\right)\right]^{\mathrm{vir}}=$ $\left[\overline{\mathcal{M}_{0, n}}(X, \beta)\right]^{\text {vir }}$ in the Chow group.

As a corollary, if $X$ is an arbitrary projective manifold and $Z$ is a collection of points, then the equality of virtual classes holds. The case where $X$ is a convex manifold and $Z$ is a collection of points has been proved in Gathmann [9]. We remark that when $g>0$ and $Z$ is a point, in general we have $\varphi_{*}\left[\overline{\mathcal{M}_{g, n}}\left(\mathrm{Bl}_{Z} X, \pi^{!} \beta\right)\right]^{\text {vir }} \neq\left[\overline{\mathcal{M}_{g, n}}(X, \beta)\right]^{\text {vir }}$. The second part of this paper is a vanishing theorem. First we introduce some notation.

- $[n]:=\{1,2, \cdots, n\}$.

- Given $A \subset[n]$, use $\overrightarrow{\tau_{\bullet} \alpha_{A}}$ to denote descendant insertions $\left\{\tau_{i_{a}} \cdot \alpha_{a}\right\}_{a \in A}$, where $\alpha_{a} \in H^{*}(X)$ and $i_{a} \geq 0$. If $i_{a}=0$ for all $a \in A$, then $\overrightarrow{\tau_{\bullet} \alpha_{A}}$ is simply denoted by $\overrightarrow{\alpha_{A}}$.

- $\overrightarrow{\mathbf{1}_{[n]}}:=(1,1, \cdots, 1)$, where $1 \in H^{*}(X)$.

- The product $\overrightarrow{\tau_{\bullet} \alpha_{A}} \cdot \overrightarrow{\tau_{\bullet} \gamma_{B}}:=\left\{\tau_{i_{a}+j_{b}} \cdot \alpha_{a} \cap \gamma_{b}\right\}_{a=b \in A \cap B} \cup\left\{\tau_{i_{a}} \cdot \alpha_{a}\right\}_{a \in A-B} \cup$ $\left\{\tau_{j_{b}} \cdot \gamma_{b}\right\}_{b \in B-A}$.

- The GW-invariant $\left\langle\overrightarrow{\tau_{\bullet} \alpha_{A}} \cdot \overrightarrow{\mathbf{1}_{[n]}}\right\rangle_{0, n, \beta}^{X}$ is simply denoted by $\left\langle\overrightarrow{\tau_{\bullet} \alpha_{A}}\right\rangle_{0, n, \beta}^{X}$. 
Theorem 1.7 Let $I, J, K$ be disjoint sets with $J \subset[n]$. Suppose $Z=\left(\bigsqcup_{i \in I} Z_{i}\right) \cup$ $\left(\bigsqcup_{j \in J} Z_{j}\right) \cup\left(\bigsqcup_{k \in K} Z_{k}\right)$ is a disjoint union of submanifolds in $X$, with the following assumptions:

- For each $i \in I \cup J, Z_{i} \subset X$ is either of type I or of type II.

- For each $k \in K, N_{Z_{k} / X}$ is convex.

- The curve class $\widetilde{\beta}=\pi^{!} \beta+\sum_{i \in I} d_{i} e_{i}+\sum_{j \in J} d_{j} e_{j}+\sum_{k \in K} d_{k} e_{k}$ with $d_{i} \neq 0$ for all $i \in I$, and $0 \neq \beta \in H_{2}(X)$. Here $e_{\bullet}$ are the exceptional line classes.

- $\overrightarrow{\omega_{J}}$ is a collection of cohomology classes in $H^{*}(\tilde{X})$. And $\operatorname{PD} \tilde{X}\left(\omega_{j}\right)$ lies in the image of $H_{*}\left(E_{j}\right) \rightarrow H_{*}(\tilde{X})$, where $E_{j}$ is the exceptional divisor, and PD means Poincaré dual.

For $i \in I \cup J$, define

$$
\delta_{i}= \begin{cases}\operatorname{rk}(\mathcal{F})-1 & \text { if } Z_{i} \subset X \text { is of type } I, \\ & \text { and } \mathcal{F} \subset N_{Z_{i} / X} \text { is generated by global sections. } \\ \operatorname{rk}\left(N_{Z_{i} / X}\right)-1 & \text { if } Z_{i} \subset X \text { is of type II. }\end{cases}
$$

Then

$\left\langle\overrightarrow{\pi^{*} \alpha_{A}} \cdot \overrightarrow{\tau_{\bullet} \gamma_{[n]}} \cdot \overrightarrow{\omega_{J}}\right\rangle_{0, n, \tilde{\beta}}^{\tilde{X}}=0$ when $\operatorname{deg} \overrightarrow{\alpha_{A}}>2\left(\operatorname{vdim}_{\mathbb{C}} \overline{\mathcal{M}}_{0, A}(X, \beta)-\sum_{i \in I} \delta_{i}-\sum_{j \in J} \delta_{j}\right)$

Here $\overrightarrow{\alpha_{A}}$ is a collection of cohomology classes from $X$ with $A \subset[n]$, and $\overrightarrow{\tau_{\bullet} \gamma_{[n]}}$ are arbitrary descendant insertions of $\tilde{X}$.

Roughly speaking, when taking $J=\varnothing$, Theorem 1.7 can be numerically interpreted as:

The image of $\varphi: \overline{\mathcal{M}}_{0, n}(\tilde{X}, \tilde{\beta}) \rightarrow \overline{\mathcal{M}}_{0, A}(X, \beta)$ has "virtual codimension" $\geq \sum_{i \in I} \delta_{i}$.

Therefore, if there are too many cohomology insertions from $X$, then the GW-invariant of $\tilde{X}$ vanishes. In [8], Gathmann proved a vanishing theorem for genus-0 nondescendant GW-invariants when blowing up at points. Theorem 1.7 is a generalization of Gathmann's results in two aspects:

(1) There is no restriction on $\operatorname{dim} Z$.

(2) Theorem 1.7 also holds for descendant GW-invariants.

We remark that Theorem 1.7 only holds for blow-ups with convex normal bundles, but does not hold for arbitrary blow-ups (see Example 5.15). 
In Example 5.13, we use Theorem 1.7 to show that, given any algebraic surface $S$ which is not (birationally equivalent to) a ruled or rational surface, then most genus -0 descendant GW-invariants of $S$ are zero. When the arithmetic genus $p_{g}(S)>0$, this conclusion has been deduced from the Image Localization Theorem of holomorphic two forms in Lee and Parker [20].

The tools used in this paper are: the degeneration formula (see Ionel and Parker [15], A-M Li and Ruan [21], J Li [22] and Maulik and Pandharipande [26]), compatibility of perfect obstruction theories (see Definition 3.3 and Behrend and Fantechi [2], Kim, Kresch and Pantev [17] and J Li and Tian [23]) and deformation invariance of virtual classes. Since there is no assumption on the manifold $X$, the moduli of stable maps of $X$ can be highly singular. Instead of analyzing singularities of the moduli space (which is nearly impossible), in Section 3 we show that if $N_{Z / X}$ is convex, then $\overline{\mathcal{M}}_{0, n}(\tilde{X}, \widetilde{\beta}) \rightarrow \overline{\mathcal{M}}_{0, n}\left(X, \pi_{*} \widetilde{\beta}\right)$ have compatible perfect obstruction theories. General blow-ups don't have this property. We use Proposition 3.15 as a criterion for the equality of (pushforward) virtual classes.

To prove Theorem 1.6, we deform the submanifold $Z$ so that the technical assumption in Proposition 3.15 is satisfied. Regarding the type I case in Theorem 1.5, a degeneration formula (in cycle forms) is used to split the problem into various relative virtual classes associated to a ruled variety $\mathbb{P}_{Z}\left(N_{Z / X} \oplus \mathcal{O}_{Z}\right)$, and then the submanifold $Z$ is moved so that the technical assumption in Proposition 3.15 is satisfied. For type II case in Theorem 1.5, we move holomorphic curves instead of $Z$ and argue directly. Although one can always move holomorphic curves as long as $N_{Z / X}$ is convex, there is a technical difficulty in applying Proposition 3.15 due to singularities of the moduli space. See Remark 4.15 for discussion.

Our starting point for the vanishing theorem is Lemma 5.1, which also requires compatible perfect obstruction theories, and therefore doesn't hold for arbitrary blow-ups. The bound of the degree of cohomology insertions in Theorem 1.7 is deduced from codimension analysis of the image on virtual normal cones.

When $N_{Z / X}$ is a direct sum of convex and concave bundles, in general we have

$$
\left\langle\pi^{*} \alpha_{1}, \cdots, \pi^{*} \alpha_{n}\right\rangle_{0, n, \pi ! \beta}^{\tilde{X}} \neq\left\langle\alpha_{1}, \cdots, \alpha_{n}\right\rangle_{0, n, \beta}^{X}
$$

The correction term will be discussed in the future.

Acknowledgements I would like to thank Harry Tamvakis for teaching me GromovWitten theory, Bong Lian for kindly suggesting me to consider virtual classes of blow-up at points, and Daniel Ruberman for his support during the course of this work. I also would like to thank Dan Abramovich and Jun Li for helpful conversations. 


\section{Preliminaries and notation}

Given a projective manifold $X$ and a curve class $\beta \in H_{2}(X)$, the stable maps moduli $\overline{\mathcal{M}}_{g, n}(X, \beta)$ collects all holomorphic maps from a genus $-g$ nodal curve with $n$ marked points $f: C \rightarrow X$. These holomorphic maps are required to satisfy the stability condition, which means the automorphism of each map is finite. Let $\mathcal{C}:=\overline{\mathcal{M}}_{g, n+1}(X, \beta)$ be the universal curve of $M:=\overline{\mathcal{M}}_{g, n}(X, \beta)$. Recall that the perfect tangent obstruction complex of $\overline{\mathcal{M}}_{g, n}(X, \beta)$ is given by Li and Tian [23]

$$
\mathcal{F}_{\bullet}=\left[\mathcal{F}_{1} \rightarrow \mathcal{F}_{2}\right]=\left[\mathcal{E} x t_{\mathcal{C} / M}^{\bullet}\left(\left[f^{*} \Omega_{X} \rightarrow \Omega_{\mathcal{C} / M}(D)\right], \mathcal{O}_{\mathcal{C}}\right)\right]
$$

where $f: \mathcal{C} \rightarrow X$ is the universal map and $D$ are the marked sections of $\overline{\mathcal{M}}_{g, n}(X, \beta)$. One also has:

(1) An evaluation map ev: $\overline{\mathcal{M}}_{g, n}(X, \beta) \rightarrow X^{n}$, which evaluates at the marked points.

(2) A line bundle $\mathbb{L}_{i}$ with the fiber over $\left(C, a_{1}, \cdots, a_{n}, f\right)$ isomorphic to the cotangent space of $C$ at $a_{i}$.

Let $\psi_{i}$ be the first Chern class $c_{1}\left(\mathbb{L}_{i}\right)$. Given $\gamma_{i} \in H^{*}(X)$, for $i=1, \cdots, n$, the genus $-g$ descendant Gromov-Witten invariants are defined as:

$$
\left\langle\tau_{a_{1}} \gamma_{1}, \cdots, \tau_{a_{n}} \gamma_{n}\right\rangle_{g, n, \beta}^{X}=\int_{\left[\overline{\mathcal{M}_{g, n}}(X, \beta)\right]^{\mathrm{vir}}} \psi_{1}^{a_{1}} \cap \cdots \cap \psi_{n}^{a_{n}} \cap \mathrm{ev}^{*}\left(\otimes_{i=1}^{n} \gamma_{i}\right) .
$$

Suppose $V$ is a vector bundle over $X$. Consider the universal family:

$$
\begin{gathered}
\overline{\mathcal{M}_{g, n+1}}(X, \beta) \stackrel{e_{n+1}}{\longrightarrow} X \\
\downarrow^{\pi_{n+1}} \\
\overline{\mathcal{M}_{g, n}}(X, \beta)
\end{gathered}
$$

$\left(R \pi_{n+1}\right)_{*} \circ e_{n+1}^{*}(V)$ can be represented by a two-term complex of vector bundles $\left[V_{0} \rightarrow V_{1}\right]$. If $\mathbf{c}$ is an invertible multiplicative characteristic class, the twisted genus $-g$ descendant Gromov-Witten invariants defined in Coates and Givental [4] are given by: $\left\langle\tau_{a_{1}} \gamma_{1}, \cdots, \tau_{a_{n}} \gamma_{n}\right\rangle_{g, n, \beta}^{X, \mathbf{c}, V}=\int_{\left[\overline{\mathcal{M}_{g, n}}(X, \beta)\right]^{\mathrm{vir}}} \psi_{1}^{a_{1}} \cap \cdots \cap \psi_{n}^{a_{n}} \cap \operatorname{ev}^{*}\left(\otimes_{i=1}^{n} \gamma_{i}\right) \cap \mathbf{c}\left(V_{0} \ominus V_{1}\right)$ 


\section{Blow-ups with convex normal bundles}

\subsection{Compatibility of perfect obstruction theories}

Given a morphism $\pi: Y \rightarrow X$ of two projective manifolds and $\widetilde{\beta} \in H_{2}(Y)$, there always exists an induced map $\varphi: \overline{\mathcal{M}}_{g, n}(Y, \widetilde{\beta}) \rightarrow \overline{\mathcal{M}}_{g, n}\left(X, \pi_{*} \widetilde{\beta}\right)$, as long as $\overline{\mathcal{M}}_{g, n}\left(X, \pi_{*} \widetilde{\beta}\right)$ makes sense (this is equivalent to saying $n \geq 3$ if $\pi_{*} \widetilde{\beta}=0$ ). If $\mathcal{E}_{\bullet}=\left[\mathcal{E}_{1} \rightarrow \mathcal{E}_{2}\right]$ and $\mathcal{F}_{\bullet}=\left[\mathcal{F}_{1} \rightarrow \mathcal{F}_{2}\right]$ are the perfect tangent-obstruction complexes on $\overline{\mathcal{M}}_{g, n}(Y, \widetilde{\beta})$ and $\overline{\mathcal{M}}_{g, n}\left(X, \pi_{*} \widetilde{\beta}\right)$ respectively, then there always exists a natural map $\mathcal{E}_{\bullet} \rightarrow \varphi^{*} \mathcal{F}_{\bullet}$ in $\mathcal{D}\left(\mathcal{O}_{\overline{\mathcal{M}}_{g, n}(Y, \widetilde{\beta})}\right)$, the derived category of the coherent sheaves on $\overline{\mathcal{M}}_{g, n}(Y, \widetilde{\beta})$. The obstruction sheaves of $\mathcal{E}_{\bullet}$ on $\overline{\mathcal{M}}_{g, n}(Y, \widetilde{\beta})$ and $\mathcal{F}_{\bullet}$ on $\overline{\mathcal{M}}_{g, n}\left(X, \pi_{*} \widetilde{\beta}\right)$ are defined as $\mathrm{Ob}_{\overline{\mathcal{M}}_{g, n}(Y, \widetilde{\beta})}:=h^{2}\left(\mathcal{E}_{\bullet}\right)$ and $\mathrm{Ob}_{\overline{\mathcal{M}}_{g, n}\left(X, \pi_{*} \tilde{\beta}\right)}:=h^{2}\left(\mathcal{F}_{\bullet}\right)$. There is a natural map $\mathrm{Ob}_{\overline{\mathcal{M}}_{g, n}(Y, \widetilde{\beta})} \rightarrow \varphi^{*}\left(\mathrm{Ob}_{\overline{\mathcal{M}}_{g, n}\left(X, \pi_{*} \widetilde{\beta}\right)}\right)$.

Suppose we have a stable map $\widetilde{p}=(\tilde{C}, \underline{a}, \tilde{f}) \in \overline{\mathcal{M}}_{g, n}(Y, \widetilde{\beta})$, where $\widetilde{C}$ is a nodal curve and $\underline{\tilde{a}} \subset \widetilde{C}$ are the marked points. The composition $\pi \circ \widetilde{f}:(\widetilde{C}, \underline{a}) \rightarrow X$ might not be stable. One contracts the unstable components to obtain the domain curve $C$. Then $\varphi(\widetilde{p}) \in \overline{\mathcal{M}}_{g, n}\left(X, \pi_{*} \widetilde{\beta}\right)$ is given by $(C, \underline{a}, f)$, where $\underline{a} \subset C$ are the marked points after contraction. We have the commutative diagram:

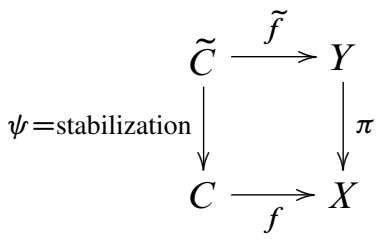

Lemma 3.1 There are canonical isomorphisms:

(1) $H^{0}\left(C, f^{*} T X\right) \cong H^{0}\left(\widetilde{C}, \tilde{f}^{*} \pi^{*} T X\right)$

(2) $H^{1}\left(C, f^{*} T X\right) \cong H^{1}\left(\widetilde{C}, \tilde{f}^{*} \pi^{*} T X\right)$.

Proof Consider a sequence of maps

$$
\widetilde{C} \stackrel{\psi}{\rightarrow} C \stackrel{\mathrm{pt}}{\rightarrow} \text { point }
$$

Set $\mathcal{F}=f^{*} T X$. Then there is a natural morphism $\mathcal{F} \rightarrow R \psi_{*} \circ L \psi^{*}(\mathcal{F})$. For any connected component $C_{i}$ of $C_{\text {unstab }}$, it must be a genus -0 nodal curve. Therefore

$$
H^{0}\left(C_{i}, \mathcal{O}_{C_{i}}\right)=\mathbb{C} \text { and } H^{j}\left(C_{i}, \mathcal{O}_{C_{i}}\right)=0 \text {, for } j \neq 0 .
$$

This implies $\mathcal{F} \rightarrow R \psi_{*} \circ L \psi^{*}(\mathcal{F})$ is an isomorphism. Since $\widetilde{C}$ and $C$ are proper, we have $\psi_{!}=\psi_{*}$ and $\mathrm{pt}_{!}=\mathrm{pt}_{*}$. Therefore

$R \mathrm{pt}_{*}(\mathcal{F}) \rightarrow R \mathrm{pt}_{*} \circ R \psi_{*} \circ L \psi^{*}(\mathcal{F})=R \mathrm{pt}_{!} \circ R \psi_{!} \circ L \psi^{*}(\mathcal{F}) \cong R\left(\mathrm{pt}^{\circ} \circ \psi\right) \circ L \psi^{*}(\mathcal{F})$ 
is an isomorphism. The lemma follows from the cohomology of this isomorphism.

Let $\pi: \tilde{X}:=\mathrm{Bl}_{Z} X \rightarrow X$ be the blow-up of $X$ along $Z \subset X$. When $g=0$ and the normal bundle $N_{Z / X}$ is convex, we have surjectivity between obstruction sheaves.

Proposition 3.2 If $N_{Z / X}$ is convex, then the natural map

$$
\mathrm{Ob}_{\overline{\mathcal{M}}_{0, n}(\tilde{X}, \widetilde{\beta})} \rightarrow \varphi^{*}\left(\mathrm{Ob}_{\overline{\mathcal{M}}_{0, n}\left(X, \pi_{*} \widetilde{\beta}\right)}\right)
$$

is surjective, where $\tilde{\beta} \in H_{2}(\tilde{X})$.

Proof For convenience, denote $\overline{\mathcal{M}}_{0, n}(\tilde{X}, \tilde{\beta})$ by $\tilde{M}$ and $\overline{\mathcal{M}}_{0, n}\left(X, \pi_{*} \tilde{\beta}\right)$ by $M$. Given a point $\tilde{p}=(\tilde{C}, \tilde{a}, \tilde{f}) \in \tilde{M}$, the obstruction space is $\left(\mathrm{Ob}_{\tilde{M}}\right)_{\tilde{p}}=h^{2}\left(\mathcal{E}_{\bullet} \otimes_{\mathcal{O}_{\tilde{M}}} k(\tilde{p})\right)$, where $k$ refers to the residue field. We also have $\left(\mathrm{Ob}_{M}\right)_{p}=h^{2}\left(\mathcal{F}_{\bullet} \otimes_{\mathcal{O}_{M}} k(p)\right)$, where $p:=\varphi(\widetilde{p})=(C, \underline{a}, f) \in M$. Consider the following commutative diagram of the right exact sequence (see Lemma 3.1):

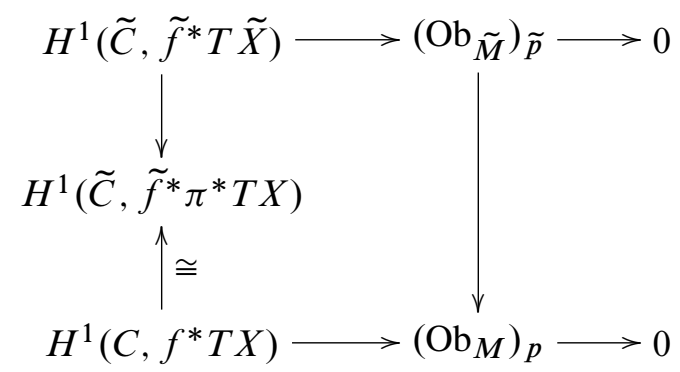

It suffices to prove

$$
H^{1}\left(\tilde{C}, \tilde{f}^{*} T \tilde{X}\right) \rightarrow H^{1}\left(\tilde{C}, \tilde{f}^{*} \pi^{*} T X\right) \text { is surjective. }
$$

First we pull back the blow-up exact sequence [5, Lemma 15.4]

$$
0 \rightarrow T \tilde{X} \rightarrow \pi^{*} T X \rightarrow i_{*} Q \rightarrow 0
$$

to $\widetilde{C}$, where $\mathrm{Q}$ is the universal quotient bundle on the exceptional divisor $E=$ $\mathbb{P}\left(N_{Z / X}\right)$ :

$$
\tilde{f}^{*} T \tilde{X} \rightarrow \tilde{f}^{*} \pi^{*} T X \rightarrow \tilde{f}^{*} Q \rightarrow 0 .
$$

And let $K_{1}$ and $K_{2}$ be the corresponding kernels:

$$
\begin{gathered}
0 \rightarrow K_{1} \rightarrow \tilde{f}^{*} \pi^{*} T X \rightarrow \tilde{f}^{*} Q \rightarrow 0 \\
0 \rightarrow K_{2} \rightarrow \tilde{f}^{*} T \tilde{X} \rightarrow K_{1} \rightarrow 0
\end{gathered}
$$


Since the domain curve has dimension $=1, H^{2}\left(\widetilde{C}, K_{2}\right)=0$, which implies

$$
H^{1}\left(\widetilde{C}, \tilde{f}^{*} T \tilde{X}\right) \rightarrow H^{1}\left(\widetilde{C}, K_{1}\right) \text { is surjective. }
$$

If we show $H^{1}\left(\tilde{C}, \tilde{f}^{*} Q\right)=0$, then the composition $H^{1}\left(\tilde{C}, \tilde{f}^{*} T \tilde{X}\right) \rightarrow H^{1}\left(\tilde{C}, K_{1}\right) \rightarrow$ $H^{1}\left(\widetilde{C}, \tilde{f}^{*} \pi^{*} T X\right)$ is also surjective. Set $C^{\prime}:=\tilde{f}^{-1}(E)$, then $H^{1}\left(\widetilde{C}, \tilde{f}^{*} Q\right)=$ $H^{1}\left(C^{\prime}, \tilde{f}^{*} Q\right)$. Note $C^{\prime}$ might be disconnected.

There is another exact sequence on the exceptional divisor $E$

$$
0 \rightarrow \mathcal{O}_{N_{Z / X}}(-1) \rightarrow \pi^{*}\left(N_{Z / X}\right) \rightarrow Q \rightarrow 0, \text { where } \pi: E \rightarrow Z .
$$

Pull it back to $C^{\prime}$ to deduce the right exact cohomology sequence

$$
H^{1}\left(C^{\prime}, \tilde{f}^{*} \pi^{*}\left(N_{Z / X}\right)\right) \rightarrow H^{1}\left(C^{\prime}, \tilde{f}^{*} Q\right) \rightarrow 0 .
$$

Note that $C^{\prime}$ is a collection of points and genus -0 nodal curves, and $N_{Z / X}$ is convex, thus we have $H^{1}\left(C^{\prime}, \tilde{f}^{*} \pi^{*}\left(N_{Z / X}\right)\right)=0$. This implies $H^{1}\left(C^{\prime}, \tilde{f}^{*} Q\right)=0$ and completes the proof.

In Behrend and Fantechi [2] and $\mathrm{Li}$ and Tian [23], the existence of global vector bundles is used to construct virtual fundamental classes. This technical assumption has been removed due to the work of A Kresch [18; 19]. Nevertheless, for simplicity, in this paper we still assume the existence of global vector bundles, which is true in Gromov-Witten Theory (see Behrend [1] and again Li and Tian [23]). In other words, $\mathcal{E}_{1}, \mathcal{E}_{2}, \mathcal{F}_{1}$ and $\mathcal{F}_{2}$ are global vector bundles, where $\mathcal{E}_{\bullet}=\left[\mathcal{E}_{1} \rightarrow \mathcal{E}_{2}\right]$ and $\mathcal{F}_{\bullet}=\left[\mathcal{F}_{1} \rightarrow \mathcal{F}_{2}\right]$ are the standard perfect obstruction theories.

First we recall the notion of compatible perfect obstruction theories $[23 ; 2 ; 17 ; 22]$ :

Definition 3.3 Suppose $\varphi$ : $\mathbf{M} \rightarrow \mathbf{N}$ is a morphism between separated Deligne-Mumford stacks. Let $\mathcal{E}^{\bullet}, \mathcal{F}^{\bullet}$ and $\mathcal{L}^{\bullet}$ be the (dual) perfect obstruction theories for $\mathbf{M}, \mathbf{N}$ and $\mathbf{M} / \mathbf{N}$. We say $\mathcal{E}^{\bullet}, \mathcal{F}^{\bullet}$ and $\mathcal{L}^{\bullet}$ are compatible if and only if we have a morphism of distinguished triangles (the bottom row is the triangle of cotangent complexes):

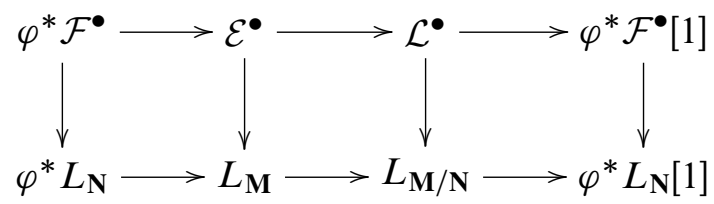

Remark 3.4 There are different versions of compatibility. One version [23; $;$; 17] requires $\mathcal{L}^{\bullet}$ must come from the relative cotangent complex associated to a local complete intersection morphism of relative Deligne-Mumford type. Here we adapt a broader definition, as used in [22]. 
Proposition 3.2 implies the existence of a relative perfect obstruction theory on $\tilde{M}=$ $\overline{\mathcal{M}}_{0, n}(\tilde{X}, \tilde{\beta}) \rightarrow M=\overline{\mathcal{M}}_{0, n}\left(X, \pi_{*} \tilde{\beta}\right)$.

Lemma 3.5 If $N_{Z / X}$ is convex, then there exists a distinguished triangle in $\mathcal{D}\left(\mathcal{O}_{\tilde{M}}\right)$

$$
\begin{gathered}
\mathcal{L}_{\bullet} \rightarrow \mathcal{E}_{\bullet} \rightarrow \varphi^{*} \mathcal{F}_{\bullet} \rightarrow \mathcal{L}_{\bullet}[1] \\
\mathcal{L}_{\bullet}=\left[\mathcal{L}_{1} \rightarrow \mathcal{L}_{2}\right], \text { where } \mathcal{L}_{i} \text { are locally free sheaves. }
\end{gathered}
$$

Proof One can always find $\mathcal{L}_{\bullet}$ so that $\mathcal{L}_{\bullet} \rightarrow \mathcal{E}_{\bullet} \rightarrow \varphi^{*} \mathcal{F}_{\bullet} \rightarrow \mathcal{L}_{\bullet}[1]$ is a distinguished triangle. Since $\tilde{M}$ has enough locally free sheaves [10], we may assume $\mathcal{L}_{\bullet}=\left[\mathcal{L}_{1} \rightarrow\right.$ $\left.\mathcal{L}_{2} \rightarrow \mathcal{L}_{3}\right]$, where $\mathcal{L}_{i}$ are locally free sheaves. The associated cohomology long exact sequence is

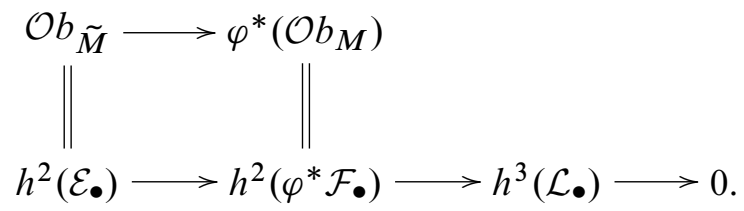

By Proposition 3.2, we know $h^{3}\left(\mathcal{L}_{\bullet}\right)=0$. This implies

$$
\tau_{\leq 2}\left(\mathcal{L}_{\bullet}\right) \rightarrow \mathcal{L}_{\bullet} \text { is quasi-isomorphic, where } \tau_{\leq 2}\left(\mathcal{L}_{\bullet}\right)=\left[\mathcal{L}_{1} \rightarrow K_{2}\right]
$$

with the short exact sequence of sheaves

$$
0 \rightarrow K_{2} \rightarrow \mathcal{L}_{2} \rightarrow \mathcal{L}_{3} \rightarrow 0 .
$$

Because $\mathcal{L}_{2}$ and $\mathcal{L}_{3}$ are locally free, $K_{2}$ is locally free as well. Replace $\mathcal{L} \bullet$ by $\tau_{\leq 2}(\mathcal{L} \bullet)$ and change the arrows accordingly, this completes the proof.

Proposition 3.6 $\mathcal{L} \in$ introduced in Lemma 3.5 gives rise to compatible perfect obstruction theories on $\varphi: \tilde{M} \rightarrow M$.

Proof Define

$$
\mathcal{L}^{\bullet}:=\left(\mathcal{L}_{\bullet}\right)^{\vee}[-1], \quad \mathcal{E}^{\bullet}:=\left(\mathcal{E}_{\bullet}\right)^{\vee}[-1], \quad \mathcal{F}^{\bullet}:=\left(\mathcal{F}_{\bullet}\right)^{\vee}[-1] .
$$

Note $\mathcal{E}^{\bullet}$ and $\mathcal{F}^{\bullet}$ are the perfect obstruction theories used in [1] and [2]. We have a distinguished triangle

$$
\varphi^{*} \mathcal{F}^{\bullet} \rightarrow \mathcal{E}^{\bullet} \rightarrow \mathcal{L}^{\bullet} \rightarrow \varphi^{*} \mathcal{F}^{\bullet}[1]
$$

We also have a distinguished triangle of cotangent complexes

$$
\varphi^{*} L_{M} \rightarrow L_{\tilde{M}} \rightarrow L_{\tilde{M} / M} \rightarrow \varphi^{*} L_{M}[1] .
$$


By the axiom of derived categories, we have a morphism of distinguished triangles:

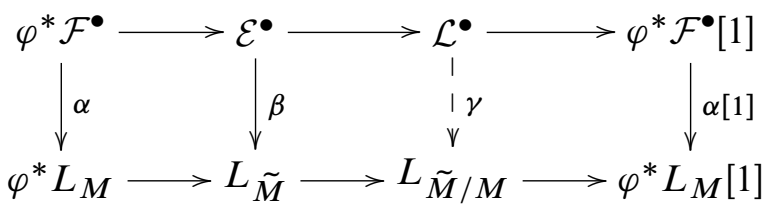

Take the associated cohomology long exact sequences of this diagram, we obtain:

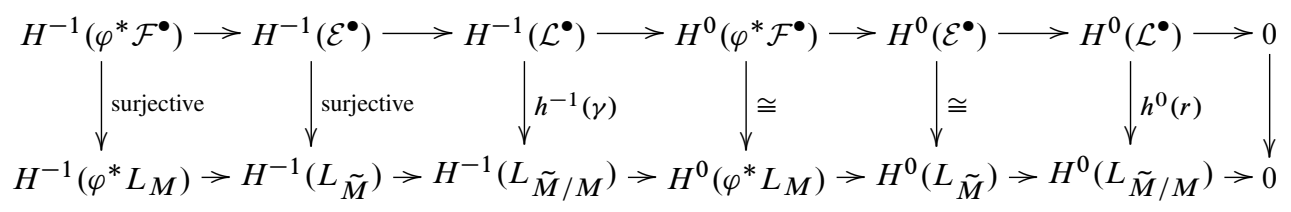

By diagram chasing, we know $h^{-1}(\gamma)$ is surjective and $h^{0}(\gamma)$ is an isomorphism. $\square$

Lemma 3.7 Suppose $\mathcal{A}, \mathcal{B}, \mathcal{C}$ are separated DM-stacks equipped with perfect obstruction theories. If $f: \mathcal{A} \rightarrow \mathcal{B}$ and $g: \mathcal{B} \rightarrow \mathcal{C}$ both have compatible perfect obstruction theories, then so does the composition map $g \circ f: \mathcal{A} \rightarrow \mathcal{C}$.

Proof This is a consequence of the octahedron axiom.

\subsection{Comparison of virtual classes}

In this section, we assume $\varphi: \mathbf{M} \rightarrow \mathbf{N}$ is a morphism between separated DeligneMumford stacks. All results will be applied to the case $\mathbf{M}=\overline{\mathcal{M}}_{0, n}\left(\tilde{X}, \pi^{!} \beta\right)$ and $\mathbf{N}=\overline{\mathcal{M}}_{0, n}(X, \beta)$, where $\beta \in H_{2}(X)$. Note that $\pi_{*} \pi^{!} \beta=\beta$.

There are two equivalent approaches to virtual classes $[2 ; 23 ; 17]$. On the other hand, when $\varphi: \mathbf{M} \rightarrow \mathbf{N}$ have compatible perfect obstruction theories, there is also a different construction of the virtual class of $\mathbf{M}$, as shown in [22, Section 4.1]. The main argument is the associativity of Gysin maps. We will quote [22, Lemma 4.3] in the following situation:

Proposition 3.8 Given a morphism $\varphi: \mathbf{M} \rightarrow \mathbf{N}$ of separated Deligne-Mumford stacks, if $\varphi$ induces compatible perfect obstruction theories, then one can construct a class $[\mathbf{M}, \mathbf{N}]^{\mathrm{vir}}$ in $A_{*}(\mathbf{M})$, and we have $[\mathbf{M}]^{\mathrm{vir}}=[\mathbf{M}, \mathbf{N}]^{\mathrm{vir}}$ in $A_{*}(\mathbf{M})$.

Suppose $\mathcal{L}_{\bullet}, \mathcal{E}_{\bullet}$ and $\mathcal{F}_{\bullet}$ are compatible perfect obstruction theories on $\mathbf{M} / \mathbf{N}, \mathbf{M}$ and $\mathbf{N}$ respectively. Now we fix notation in the construction of $[\mathbf{M}, \mathbf{N}]^{\mathrm{vir}}$. Define $\mathrm{Ob}_{\mathbf{M} / \mathbf{N}}:=h^{2}\left(\mathcal{L}_{\bullet}\right)$ as the relative obstruction sheaf. There is an infinitesimal model 
(denoted by $\left\{\mathcal{D}(p)_{2}\right\}_{p \in \mathbf{M}}$ in [22]) over the pair $\left(\mathbf{M}, \mathrm{Ob}_{\mathbf{M} / \mathbf{N}} \oplus \varphi^{*} \mathrm{Ob}_{\mathbf{N}}\right)$. Consider the surjective map

$$
\mathcal{L}_{2} \oplus \varphi^{*} \mathcal{F}_{2} \rightarrow \mathrm{Ob}_{\mathbf{M} / \mathbf{N}} \oplus \varphi^{*} \mathrm{Ob}_{\mathbf{N}} \rightarrow 0 .
$$

This gives rise to a cone $\mathbb{C}_{\mathbf{M}} \subset \operatorname{Vect}\left(\mathcal{L}_{2} \oplus \varphi^{*} \mathcal{F}_{2}\right)$, so that $\mathbb{C}_{\mathbf{M}}$ is consistent with $\left\{\mathcal{D}(p)_{2}\right\}_{p \in \mathbf{M}}$. The second construction $[\mathbf{M}, \mathbf{N}]^{\mathrm{vir}}$ is defined as the intersection class of $\mathbb{C}_{\mathbf{M}}$ with the zero section of $\mathcal{L}_{2} \oplus \varphi^{*} \mathcal{F}_{2}$.

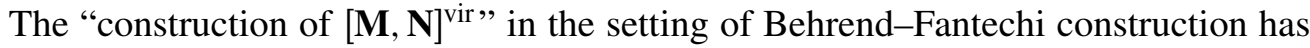
appeared in [17, Theorem 1], which is only formulated in the case where $\mathcal{L}^{\bullet}$ is the pullback of a relative cotangent complex associated to a local complete intersection morphism of relative Deligne-Mumford type. However, the second part in the proof of [17, Theorem 1] doesn't rely on "local complete intersection morphism". Therefore the proof can be slightly rearranged to give "the construction of $[\mathbf{M}, \mathbf{N}]^{\mathrm{vir}}$ " in the broader definition of compatibility (Definition 3.3). Here we briefly describe how this is achieved by the argument in [17].

Let $\mathfrak{C}_{\mathbf{N}}$ be the (intrinsic) normal cone stack of $\mathbf{N}$, and let $\mathfrak{C}_{\mathbf{M} / \mathbf{N}}$ be the relative normal

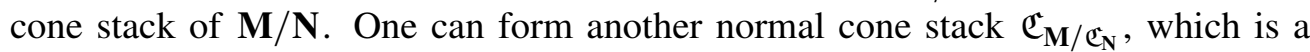
natural subcone stack of $\mathfrak{C}_{\mathbf{M} / \mathbf{N}} \times \mathbf{M} \varphi^{*} \mathfrak{C}_{\mathbf{N}}$. Therefore $\mathfrak{C}_{\mathbf{M} / \mathfrak{C}_{\mathbf{N}}}$ embeds in the vector bundle stack $\sigma: h^{2} / h^{1}\left(\mathcal{L}_{\bullet}\right) \oplus \varphi^{*}\left(h^{2} / h^{1}\left(\mathcal{F}_{\bullet}\right)\right) \rightarrow \mathbf{M}$. The new class $[\mathbf{M}, \mathbf{N}]^{\text {vir }}$ is defined as $\left(\sigma^{*}\right)^{-1}\left(\left[\mathfrak{C}_{\mathbf{M} / \mathfrak{C}_{\mathbf{N}}}\right]\right)$.

Given a morphism $X \rightarrow Y$ of relative Deligne-Mumford type, denote the deformation (to the normal cone) stack by $\mathcal{M}_{X / Y}^{0} \rightarrow \mathbb{P}^{1}$, with the fiber over $\{0\} \in \mathbb{P}^{1}$ isomorphic to the normal cone stack $\mathfrak{C}_{X / Y}$. If $Y=\operatorname{spec}(\mathbb{C})$, denote the deformation stack simply by $\mathcal{M}_{X}^{0}$. In order to show

$$
\left(\sigma^{*}\right)^{-1}\left(\left[\mathfrak{C}_{\mathbf{M} / \mathfrak{C}_{\mathbf{N}}}\right]\right)=[\mathbf{M}]^{\mathrm{vir}} \in A_{*}(\mathbf{M}),
$$

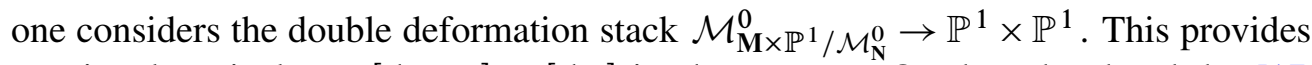

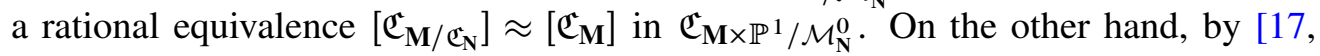

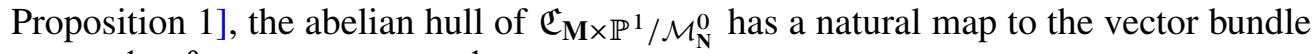
stack $h^{1} / h^{0}(c(g))$ on $\mathbf{M} \times \mathbb{P}^{1}$, where $c(g)$ is the mapping cone associated to

$$
\mathcal{E}_{\bullet} \oplus \varphi^{*} \mathcal{F}_{\bullet} \stackrel{g}{\rightarrow} \varphi^{*} \mathcal{F}_{\bullet} \otimes \mathcal{O}_{\mathbb{P}^{1}}(1) \text { on } \mathbf{M} \times \mathbb{P}^{1} .
$$

Now the rational equivalence can be pushed forward to $h^{1} / h^{0}(c(g))$. It is easy to see that the pull back of $h^{1} / h^{0}(c(g))$ to $\mathbf{M} \times\{0\}$ and $\mathbf{M} \times\{1\}$, correspond to $h^{2} / h^{1}\left(\mathcal{L}_{\bullet}\right) \oplus$ $\varphi^{*}\left(h^{2} / h^{1}\left(\mathcal{F}_{\bullet}\right)\right)$ and $h^{2} / h^{1}\left(\mathcal{E}_{\bullet}\right)$ respectively. Therefore $\left(\sigma^{*}\right)^{-1}\left(\left[\mathfrak{C}_{\mathbf{M} / \mathfrak{C}_{\mathbf{N}}}\right]\right)=[\mathbf{M}]^{\text {vir }}$.

Remark 3.9 At the beginning of [22, Section 4.1], it is assumed that $\mathbf{M} \rightarrow \mathbf{N}$ is representable. One can drop this assumption by taking a presentation of $\mathbf{M}$ : a surjective étale 
morphism from a scheme $T \rightarrow \mathbf{M}$. There are natural compatible perfect obstruction theories on $T \rightarrow \mathbf{N}$ induced from those on $\mathbf{M} \rightarrow \mathbf{N}$. Note $T \rightarrow \mathbf{N}$ is representable, so we can apply [22, Lemma 4.3]. On the other hand, the construction of various cones, cycles and rational equivalence in the proof of Lemma 4.3 are canonical, and they descend to the case $\mathbf{M} \rightarrow \mathbf{N}$. Alternatively, this can also be seen via the construction in [17], as described in the previous paragraph.

Remark 3.10 The "construction of $[\mathbf{M}, \mathbf{N}]^{\mathrm{vir}}$ " is only useful when one has a good understanding of the relative obstruction theory $\mathcal{L}^{\bullet}$, otherwise it simply transforms a problem into something unknown. In practice, it is usually quoted in the form of [17, Theorem 1], where $\mathcal{L}^{\bullet}$ comes from local complete intersection.

Regarding the construction of $[\mathbf{N}]^{\text {vir }}$, take the surjective map $\mathcal{F}_{2} \rightarrow \mathrm{Ob}_{\mathbf{N}} \rightarrow 0$.

This gives rise to a cone $\mathbb{C}_{\mathbf{N}} \subset \operatorname{Vect}\left(\mathcal{F}_{2}\right)$, so that $\mathbb{C}_{\mathbf{N}}$ is consistent with the infinitesimal model over $\left(\mathbf{N}, \mathrm{Ob}_{\mathbf{N}}\right)$. $[\mathbf{N}]^{\text {vir }}$ is defined as the intersection class of $\mathbb{C}_{\mathbf{N}}$ with the zero section of $\mathcal{F}_{2}$. Note that $\mathbb{C}_{\mathbf{M}}$ is a cone with pure dimension $=\operatorname{vdim}_{\mathbb{C}}(\mathbf{M})+\operatorname{rk}\left(\mathcal{L}_{2}\right)+$ $\operatorname{rk}\left(\mathcal{F}_{2}\right)$, and $\mathbb{C}_{\mathbf{N}}$ is a cone with pure dimension $=\operatorname{vdim}_{\mathbb{C}}(\mathbf{N})+\operatorname{rk}\left(\mathcal{F}_{2}\right)$.

Lemma 3.11 We have the following diagram (not Cartesian product):

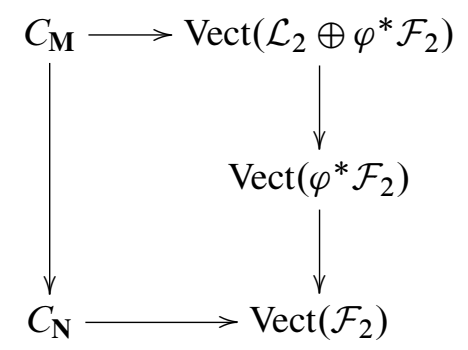

Proof The properties of $\mathbb{C}_{M}$ and $\mathbb{C}_{\mathbf{N}}$ are determined by properties of infinitesimal models, therefore it suffices to prove the corresponding diagram in the infinitesimal models, which is straightforward. An alternative way to see this is via the construction in [2] and [17].

On the other hand, $\operatorname{Vect}\left(\mathcal{L}_{2} \oplus \varphi^{*} \mathcal{F}_{2}\right)$ can be also regarded as a vector bundle over $\operatorname{Vect}\left(\varphi^{*} \mathcal{F}_{2}\right)$. Let

$$
0_{\mathcal{L}_{2}}: \operatorname{Vect}\left(\varphi^{*} \mathcal{F}_{2}\right) \rightarrow \operatorname{Vect}\left(\mathcal{L}_{2} \oplus \varphi^{*} \mathcal{F}_{2}\right)
$$


be the zero section, then we have the diagram:

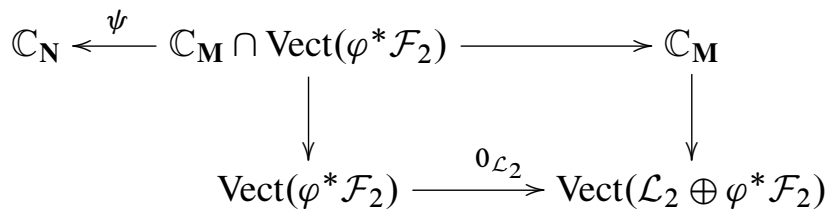

The right square is a Cartesian product, and $\psi$ is the map induced by $\mathbb{C}_{\mathbf{M}} \rightarrow \mathbb{C}_{\mathbf{N}}$ in Lemma 3.11. Note $\psi$ is proper as long as $\varphi$ is proper. We also have

$$
0_{\mathcal{L}_{2}}^{!}\left[\mathbb{C}_{\mathbf{M}}\right] \in A_{d+\operatorname{rk}\left(\mathcal{F}_{2}\right)}\left(\mathbb{C}_{\mathbf{M}} \cap \operatorname{Vect}\left(\varphi^{*} \mathcal{F}_{2}\right)\right) \text {, where } 0_{\mathcal{L}_{2}} \text { is the refined Gysin map. }
$$

In the blow-up case $\varphi: \overline{\mathcal{M}}_{0, n}\left(\tilde{X}, \pi^{!} \beta\right) \rightarrow \overline{\mathcal{M}}_{0, n}(X, \beta)$, consider the following diagram:

$$
\begin{gathered}
\overline{\mathcal{M}}_{0, n+1}(X, \beta) \stackrel{e_{n+1}}{\longrightarrow} X \\
\downarrow^{\pi_{n+1}} \\
\overline{\mathcal{M}}_{0, n}(X, \beta)
\end{gathered}
$$

Let $U$ be the complement of $\pi_{n+1}\left(e_{n+1}^{-1}(Z)\right)$ in $\overline{\mathcal{M}}_{0, n}(X, \beta)$, therefore $U$ is an open substack of $\overline{\mathcal{M}}_{0, n}(X, \beta)$. Given $(C, \underline{a}, f) \in M$, we have:

$$
(C, \underline{a}, f) \in U \Leftrightarrow f(C) \cap Z=\phi .
$$

Because $\pi: \tilde{X} \rightarrow X$ is the blow up of $X$ along $Z$, we deduce:

Lemma 3.12 There is an isomorphism $\varphi: \varphi^{-1}(U) \rightarrow U$ with the same (in the sense of quasi-isomorphic) perfect obstruction theory.

Because of the above lemma, it motivates us to analyze the following situation:

Suppose the proper morphism $\varphi: \mathbf{M} \rightarrow \mathbf{N}$ induces compatible perfect obstruction theories with $d=\operatorname{vdim}_{\mathbb{C}}(\mathbf{M})=\operatorname{vdim}_{\mathbb{C}}(\mathbf{N})$. Moreover, we assume that there exists an open substack $U$ in $\mathbf{N}$, so that $\varphi: \varphi^{-1}(U) \rightarrow U$ is an isomorphism with the same perfect obstruction theories.

Lemma 3.13 Under the setting in the previous paragraph, we have $\left.\left(\psi_{*} 0_{\mathcal{L}_{2}}\left[\mathbb{C}_{\mathbf{M}}\right]\right)\right|_{U}=\left[\left.\mathbb{C}_{\mathbf{N}}\right|_{U}\right]$ in $A_{d+\operatorname{rk}\left(\mathcal{F}_{2}\right)}\left(\left.\mathbb{C}_{\mathbf{N}}\right|_{U}\right)$, where $d=\operatorname{vdim}_{\mathbb{C}} \mathbf{M}=\operatorname{vdim}_{\mathbb{C}} \mathbf{N}$ 
Proof Because $\varphi^{-1} U \cong U$ and the flat pull back $\left.(\bullet)\right|_{U}$ commutes with other operators, we have

$$
\left.\left(\psi_{*} 0_{\mathcal{L}_{2}}\left[\mathbb{C}_{\mathbf{M}}\right]\right)\right|_{U}=\psi_{*} 0_{\mathcal{L}_{2}}\left(\left.\mathbb{C}_{\mathbf{M}}\right|_{U}\right)
$$

Moreover, because $\left.\left.\mathcal{E}_{\bullet}\right|_{\varphi^{-1}(U)} \cong \mathcal{F}_{\bullet}\right|_{U}$, we know $\left.\mathrm{Ob}_{\mathbf{M} / \mathbf{N}}\right|_{U}$ vanishes. Therefore the infinitesimal models on $\varphi^{-1}(U) \cong U$ are the same, and we have the Cartesian diagram:

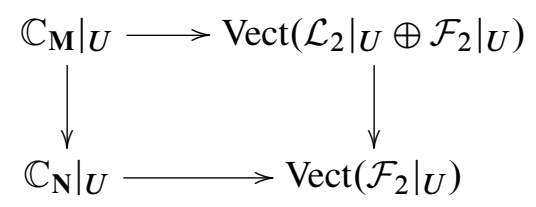

That is, $\operatorname{Vect}\left(\left.\left.\mathcal{L}_{2}\right|_{U} \oplus \mathcal{F}_{2}\right|_{U}\right)$ is a vector bundle over $\operatorname{Vect}\left(\left.\mathcal{F}_{2}\right|_{U}\right)$, and $\left.\mathbb{C}_{\mathbf{M}}\right|_{U}$ is the flat pull back of $\left.\mathbb{C}_{\mathbf{N}}\right|_{U}$. Thus we have $\psi_{*} 0_{\mathcal{L}_{2}}^{!}\left(\left.\mathbb{C}_{\mathbf{M}}\right|_{U}\right)=\left[\left.\mathbb{C}_{\mathbf{N}}\right|_{U}\right]$ in $A_{d+\operatorname{rk}\left(\mathcal{F}_{2}\right)}\left(\left.\mathbb{C}_{\mathbf{N}}\right|_{U}\right)$.

Suppose $\mathbb{C}_{\mathbf{N}}$ has irreducible components $\mathbb{C}_{i}, i=1, \cdots, k$. Let $\operatorname{supp}(\bullet)$ be the support of a cone. In the rest of this section, we will assume the open substack $U \subset \mathbf{N}$ satisfies the following technical assumption:

(Assumption $*) \quad \operatorname{supp}\left(\mathbb{C}_{i}\right) \cap U$ is nonempty in $\mathbf{N}$ for $i=1, \cdots, k$.

Because $\mathbb{C}_{\mathbf{N}}$ is a cone with pure dimension $d+\operatorname{rk}\left(\mathcal{F}_{2}\right)$, (Assumption $*$ ) implies

$$
\operatorname{dim}\left(\mathbb{C}_{\mathbf{N}}-\left(\left.\mathbb{C}_{\mathbf{N}}\right|_{U}\right)\right)<d+\operatorname{rk}\left(\mathcal{F}_{2}\right) .
$$

With this technical assumption, it is easy to prove that $\varphi: \mathbf{M} \rightarrow \mathbf{N}$ is virtually birational.

Corollary 3.14 If the open substack $U \subset \mathbf{N}$ satisfies (Assumption $*$ ), then we have

$$
\psi_{*} 0_{\mathcal{L}_{2}}\left[\mathbb{C}_{\mathbf{M}}\right]=\left[\mathbb{C}_{\mathbf{N}}\right] \text { in } A_{d+\mathrm{rk}\left(\mathcal{F}_{2}\right)}\left(\mathbb{C}_{\mathbf{N}}\right)
$$

Proof We have

$$
\operatorname{dim}\left(\mathbb{C}_{\mathbf{N}}-\left(\left.\mathbb{C}_{\mathbf{N}}\right|_{U}\right)\right)<d+\operatorname{rk}\left(\mathcal{F}_{2}\right) \Longrightarrow A_{d+\operatorname{rk}\left(\mathcal{F}_{2}\right)}\left(\mathbb{C}_{\mathbf{N}}-\left.\mathbb{C}_{\mathbf{N}}\right|_{U}\right)=0
$$

Combined with the right exact sequence:

$$
A_{d+\mathrm{rk}\left(\mathcal{F}_{2}\right)}\left(\mathbb{C}_{\mathbf{N}}-\left.\mathbb{C}_{\mathbf{N}}\right|_{U}\right) \rightarrow A_{d+\mathrm{rk}\left(\mathcal{F}_{2}\right)}\left(\mathbb{C}_{\mathbf{N}}\right) \rightarrow A_{d+\mathrm{rk}\left(\mathcal{F}_{2}\right)}\left(\left.\mathbb{C}_{\mathbf{N}}\right|_{U}\right) \rightarrow 0,
$$

we know $A_{d+\operatorname{rk}\left(\mathcal{F}_{2}\right)}\left(\mathbb{C}_{\mathbf{N}}\right) \cong A_{d+\operatorname{rk}\left(\mathcal{F}_{2}\right)}\left(\left.\mathbb{C}_{\mathbf{N}}\right|_{U}\right)$. By Lemma 3.13, we obtain

$$
\psi_{*} 0_{\mathcal{L}_{2}}\left[\mathbb{C}_{\mathbf{M}}\right]=\left[\mathbb{C}_{\mathbf{N}}\right] \text { in } A_{d+\mathrm{rk}\left(\mathcal{F}_{2}\right)}\left(\mathbb{C}_{\mathbf{N}}\right)
$$

Now we summarize all results in this section to deduce the following: 
Proposition 3.15 Suppose the proper morphism $\varphi: \mathbf{M} \rightarrow \mathbf{N}$ induces compatible perfect obstruction theories with $d=\operatorname{vdim}_{\mathbb{C}}(\mathbf{M})=\operatorname{vdim}_{\mathbb{C}}(\mathbf{N})$. We also assume that there exists an open substack $U$ in $\mathbf{N}$, so that $\varphi: \varphi^{-1}(U) \rightarrow U$ is an isomorphism with the same perfect obstruction theories.

If $U \cap \operatorname{supp}\left(\right.$ each irreducible component of $\mathbb{C}_{\mathbf{N}}$ ) is nonempty in $\mathbf{N}$, then $\varphi_{*}[\mathbf{M}]^{\mathrm{vir}}=$ $[\mathbf{N}]^{\text {vir }}$ in the Chow group $A_{d}(\mathbf{N})$.

Proof Recall the diagram with the right square as Cartesian product:

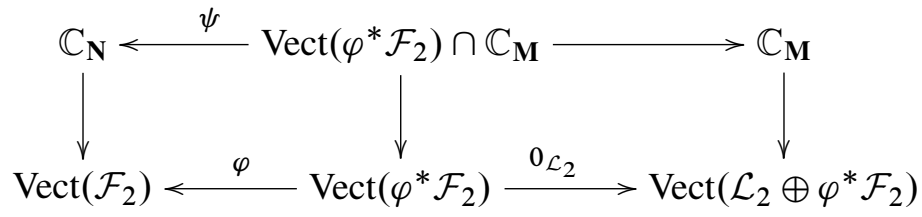

If we regard $\left[\mathbb{C}_{\mathbf{M}}\right]$ as a class in $A_{*}\left(\operatorname{Vect}\left(\mathcal{L}_{2} \oplus \varphi^{*} \mathcal{F}_{2}\right)\right)$, and $\left[\mathbb{C}_{\mathbf{N}}\right]$ as a class in $A_{*}\left(\operatorname{Vect}\left(\mathcal{F}_{2}\right)\right)$, then Corollary 3.14 implies

$$
\varphi_{*} 0_{\mathcal{L}_{2}}^{!}\left[\mathbb{C}_{\mathbf{M}}\right]=\left[\mathbb{C}_{\mathbf{N}}\right] \text { in } A_{d+\operatorname{rk}\left(\mathcal{F}_{2}\right)}\left(\operatorname{Vect}\left(\mathcal{F}_{2}\right)\right) .
$$

From another diagram with the left square as Cartesian product:

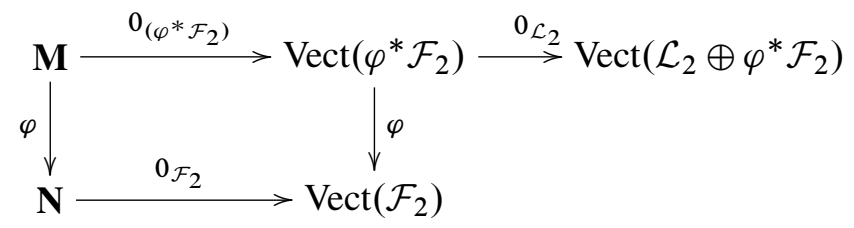

$$
\begin{aligned}
\varphi_{*}[\mathbf{M}]^{\mathrm{vir}} & =\varphi_{*} \circ 0_{\left(\varphi^{*} \mathcal{F}_{2}\right)}^{!} \circ 0_{\mathcal{L}_{2}}^{!}\left[\mathbb{C}_{\mathbf{M}}\right]=0_{\mathcal{F}_{2}}^{!} \circ \varphi_{*} \circ 0_{\mathcal{L}_{2}}^{!}\left[\mathbb{C}_{\mathbf{M}}\right] \\
& =0_{\mathcal{F}_{2}}^{!}\left[\mathbb{C}_{\mathbf{N}}\right]=[\mathbf{N}]^{\mathrm{vir}}
\end{aligned}
$$

Here we use $0{ }_{\left(\varphi^{*} \mathcal{F}_{2}\right)}^{!}=0_{\mathcal{F}_{2}}^{!}: A_{*}\left(\operatorname{Vect}\left(\varphi^{*} \mathcal{F}_{2}\right)\right) \rightarrow A_{*}(\mathbf{M})$.

Corollary 3.16 Suppose the proper morphism $\varphi: \mathbf{M} \rightarrow \mathbf{N}$ induces compatible perfect obstruction theories with $d=\operatorname{vdim}_{\mathbb{C}}(\mathbf{M})=\operatorname{vdim}_{\mathbb{C}}(\mathbf{N})$. Suppose there exists an open substack $U$ in $\mathbf{N}$, so that $\varphi(\mathbf{M}) \cap U=\varnothing$.

If $U \cap \operatorname{supp}\left(\right.$ each irreducible component of $\mathbb{C}_{\mathbf{N}}$ ) is nonempty in $\mathbf{N}$, then $\varphi_{*}[\mathbf{M}]^{\mathrm{vir}}=0$ in the Chow group $A_{d}(\mathbf{N})$.

Proof Apply the previous proposition to $\varphi \bigsqcup \mathrm{Id}: \mathbf{M} \bigsqcup \mathbf{N} \rightarrow \mathbf{N}$. 


\subsection{Transversal intersection of two manifolds}

Suppose $X$ and $Y$ are two arbitrary closed submanifolds of a compact homogeneous space $\mathcal{P}$, and $Z$ is the transversal intersection of $X$ and $Y$. Suppose the group variety $G$ acts on $\mathcal{P}$ transitively.

Lemma 3.17 The normal bundle $N_{Z / X}$ is generated by global sections, and therefore is convex.

Proof The tangent bundle $T \mathcal{P}$ is generated by global sections, and $N_{Y / \mathcal{P}}$ is a quotient bundle of $T \mathcal{P}$. This implies $N_{Y / \mathcal{P}}$ is generated by global sections as well. Note $N_{Z / X}$ is the pull back of $N_{Y / \mathcal{P}}$ to $Z$.

Consider $\pi: \tilde{X} \rightarrow X$, the blow up of $X$ along the submanifold $Z$. We may assume $\operatorname{codim}_{\mathbb{C}}(Y, \mathcal{P}) \geq 2$. The first attempt is to apply Proposition 3.15, but the technical assumption

supp(each irreducible component of $\left.\mathbb{C}_{\mathbf{N}}\right) \cap U$ is nonempty in $\mathbf{N}$,

$$
\text { where } \mathbf{N}=\overline{\mathcal{M}}_{0, n}(X, \beta)
$$

may not be satisfied. We will choose an element $\sigma \in G$, and show the technical assumption is satisfied when $\mathrm{Z}$ is perturbed to $X \cap Y^{\sigma}$.

Lemma 3.18 Given a holomorphic map from a compact curve $f: C \rightarrow \mathcal{P}$, define

$$
B_{(C, f)}:=\left\{\sigma \in G \quad \mid \quad f(C) \cap Y^{\sigma} \neq \varnothing\right\} .
$$

Then $B_{(C, f)}$ is closed in $G$, and $\operatorname{dim}\left(B_{(C, f)}\right)<\operatorname{dim} G$.

Proof Consider $G \stackrel{p_{1}}{\longleftarrow} G \times Y \stackrel{\Phi}{\longrightarrow} \mathcal{P}$, where $p_{1}$ is the projection and $\Phi$ is the group action. Note $B_{(C, f)}=p_{1}\left(\Phi^{-1}(f(C))\right)$ is closed in $G$ because $p_{1}$ is proper. Because $G$ acts on $\mathcal{P}$ transitively, $\Phi$ is a smooth morphism. Therefore,

$$
\begin{aligned}
\operatorname{dim} B_{(C, f)} & \leq \operatorname{dim} Y+\operatorname{dim} G-\operatorname{dim} \mathcal{P}+\operatorname{dim} f(C) \\
& \leq \operatorname{dim} G-\operatorname{codim}_{\mathbb{C}}(Y, \mathcal{P})+1 \leq \operatorname{dim} G-1 .
\end{aligned}
$$

Lemma 3.19 Define $W:=\left\{\sigma \in G \mid Y^{\sigma}\right.$ is not transversal to $\left.X\right\}$. Then $W$ is closed in $G$, with $\operatorname{dim} W<\operatorname{dim} G$.

Proof Note the identity element $\operatorname{Id} \in G$ does not belong to $W$. 
Suppose $\mathcal{F}_{\bullet}$ is the perfect obstruction theory on $\mathbf{N}=\overline{\mathcal{M}_{0, n}}(X, \beta)$, and the virtual normal cone $\mathbb{C}_{\mathbf{N}}$ has irreducible components $\mathbb{C}_{i}$, for $i=1, \ldots, k$. For each $i$, we pick a point $\left(C_{i}, \underline{a}_{i}, f_{i}\right) \in \operatorname{supp}\left(\mathbb{C}_{i}\right)$. By the previous two lemmas, we know

$$
W \cup\left(\bigcup_{i=1}^{k} B_{\left(C_{i}, f_{i}\right)}\right) \text { is closed in } G \text { with codimension } \geq 1 \text {. }
$$

Therefore we can take an affine smooth locally closed curve $S \hookrightarrow G$ such that:

(1) $\operatorname{Id} \in S$,

(2) $(S-\mathrm{Id}) \cap\left(\bigcup_{i=1}^{k} B_{\left(C_{i}, f_{i}\right)}\right)=\varnothing$,

(3) $Y^{\sigma}$ is transversal to $X, \forall \sigma \in S$.

Choose an element $\sigma \in S$, then $Z=X \cap Y$ is deformation equivalent to $Z_{\sigma}:=X \cap Y^{\sigma}$. Note the normal bundle $N_{Z_{\sigma} / X}$ is still generated by global sections. The technical assumption of Proposition 3.15 is satisfied for $\tilde{X} \rightarrow X$.

Lemma 3.20 We have the equality

supp(each irreducible component of $\left.\mathbb{C}_{\mathbf{N}}\right) \cap U$ is nonempty in $\mathbf{N}=\overline{\mathcal{M}_{0, n}}(X, \beta)$, where $U$ is a collection of stable maps supported away from $Z_{\sigma}$.

Proof The choice of the curve $S \hookrightarrow G$ asserts $f_{i}\left(C_{i}\right) \cap Y^{\sigma}=\varnothing$, for $i=1, \ldots, k$. Hence $\left(C_{i}, \underline{a}_{i}, f_{i}\right) \in U$.

Proposition 3.15 and deformation invariance of virtual classes implies:

Theorem 3.21 Suppose $Z$ is the transversal intersection of two manifolds $X$ and $Y$ in a compact homogeneous space $\mathcal{P}$. Then we have $\varphi_{*}\left[\overline{\mathcal{M}_{0, n}}\left(\tilde{X}, \pi^{!} \beta\right)\right]^{\mathrm{vir}}=$ $\left[\overline{\mathcal{M}_{0, n}}(X, \beta)\right]^{\text {vir }}$ in the Chow group.

The theorem can be generalized to the case when $Z$ is the intersection of $X$ with multiple manifolds. More precisely, suppose $Y_{i}, i=1, \ldots, m$, are submanifolds of a homogeneous space $P$. We assume $Y_{k+1}$ is transversal to $X \cap\left(\bigcap_{i=1}^{k} Y_{i}\right)$, for $k=0, \ldots, m-1$.

Corollary 3.22 Let $Z$ be $X \cap\left(\bigcap_{i=1}^{m} Y_{i}\right)$. Then we have

$$
\varphi_{*}\left[\overline{\mathcal{M}_{0, n}}\left(\tilde{X}, \pi^{!} \beta\right)\right]^{\mathrm{vir}}=\left[\overline{\mathcal{M}_{0, n}}(X, \beta)\right]^{\mathrm{vir}} .
$$


Proof Define $G^{\prime}:=G^{m}$ and $\mathcal{P}^{\prime}:=\mathcal{P}^{m}$. Then $\mathcal{P}^{\prime}$ is a homogeneous space with respect to the group variety $G^{\prime}$. Let $\Delta: X \rightarrow \mathcal{P}^{\prime}$ be the diagonal map. $X$ is transversal to the manifold $\prod_{i=1}^{m} Y_{i}$ in the ambient space $\mathcal{P}^{\prime}$. Apply the previous theorem to the case $X \rightarrow \mathcal{P}^{\prime}, Y:=\prod_{i=1}^{m} Y_{i}$, and $Z=X \cap Y$ in $\mathcal{P}^{\prime}$.

A similar argument also implies:

Corollary 3.23 Suppose $X$ is a projective manifold, and $Z$ is a collection of points in $X$. Then

$$
\varphi_{*}\left[\overline{\mathcal{M}_{0, n}}\left(\tilde{X}, \pi^{!} \beta\right)\right]^{\mathrm{vir}}=\left[\overline{\mathcal{M}_{0, n}}(X, \beta)\right]^{\mathrm{vir}} .
$$

Proof This is because $N_{Z / X}$ is convex, and $Z$ can always be moved.

\section{Virtual birationality after degeneration}

In the previous subsection, the submanifold is deformed so that the technical assumption in Proposition 3.15 is satisfied. In general, if $N_{Z / X}$ has a nonzero section, it doesn't imply $Z$ can be moved. The degeneration formula reduces the problem to a ruled variety, where $Z$ can be moved if $N_{Z / X}$ has a section. The degeneration formula has been clearly presented in Li [22], Graber and Vakil [11] and Liu and Yau [25]. The purpose of the first subsection is to fix notation.

\subsection{Degeneration formula from blow-ups}

$(X, D)$ is called a relative pair if $D$ is a smooth divisor of the manifold $X$. The $\mathbb{P}^{1}$-bundle $B:=\mathbb{P}_{D}\left(N_{D / X} \oplus \mathcal{O}_{D}\right)$ has two divisors: the zero divisor (with normal bundle $N_{D / X}^{\vee}$ ) and the infinity divisor (with normal bundle $N_{D / X}$ ). $B_{l}$ is defined as the union of $l$-copies of $B$, by gluing the infinity divisor of the $i$-th component to the zero divisor of the $(i+1)$-th component. Let $D_{i}$ be the zero divisor of the $(i+1)$-th component. Note $\operatorname{Sing}\left(B_{l}\right)=\bigcup_{i=1}^{l-1} D_{i}$. Define $X_{l}:=X \cup_{D_{0}} B_{l}$.

Now we recall Definition 4.6 in [22]:

Definition 4.1 An admissible weighted graph $\Gamma$ for a relative pair $(X, D)$ is a graph without edges together with the following data:

(1) An ordered collection of legs, an ordered collection of weighted roots, and two weight functions on the vertex set $g: V(\Gamma) \rightarrow \mathbb{Z}_{\geq 0}$ and $b: V(\Gamma) \rightarrow H_{2}(X)$.

(2) $\Gamma$ is relatively connected in the sense that either $|V(\Gamma)|=1$ or each vertex in $V(\Gamma)$ has at least one root attached to it. 
The weight functions $g$ and $b$ in the previous definition are used to record the genus and the homology class in $X$ for each connected component of a domain curve. Denote the moduli of relative stable maps to $(X, D)$ with topological data $\Gamma$ by $\overline{\mathcal{M}}(X, D, \Gamma)$. A $\mathbb{C}$-point in $\overline{\mathcal{M}}(X, D, \Gamma)$ is a holomorphic map $C \stackrel{f}{\rightarrow} X_{l} \rightarrow X$ satisfying stability and predeformability together with topological constraints $\Gamma$. The domain curve is disconnected if and only if $|V(\Gamma)|>1$. For convenience, define

$$
b(\Gamma):=\sum_{v \in V(\Gamma)} b(v) \text { and } g(\Gamma):=1-|V(\Gamma)|+\sum_{v \in V(\Gamma)} g(v) .
$$

Let $\mathcal{T}$ be the Artin stack parametrizing the possible target of relative stable maps to $(X, D)$. Let $\mathfrak{M}_{g, k}$ be the Artin stack of prestable genus $g$ curves with $k$ marked points. The perfect obstruction theory on $\overline{\mathcal{M}}(X, D, \Gamma)$ is induced from the relative perfect obstruction theory on

$$
\overline{\mathcal{M}}(X, D, \Gamma) \rightarrow \mathcal{T} \times \mathfrak{M}_{g(\Gamma), k}, \text { where } k=\text { number of legs }+ \text { number of roots. }
$$

Given an arbitrary manifold $X$ with a submanifold $Z$, deformation to the normal cone is obtained from the blow-up of a trivial family:

$$
W:=\mathrm{Bl}_{Z \times\{0\}} X \times \mathbb{C} \rightarrow \mathbb{C} .
$$

Note $W_{t} \cong X$ for $t \neq 0$ and $W_{0}=\tilde{X} \bigcup_{\mathbb{P}\left(N_{Z / X}\right)} \mathbb{P}\left(N_{Z / X} \oplus \mathcal{O}_{Z}\right)$, where $\pi: \tilde{X}=$ $\mathrm{Bl}_{Z} X \rightarrow X$. Denote $\mathbb{P}\left(N_{Z / X}\right)$ by $D$, and $p_{2}: \mathbb{P}\left(N_{Z / X} \oplus \mathcal{O}_{Z}\right) \rightarrow Z$.

Theorem 4.2 (Degeneration formula from blow-up [22; 25])

$\left[\overline{\mathcal{M}}\left(\mathcal{W}_{0}, g, n, \beta\right)\right]^{\mathrm{vir}}$

$$
\begin{array}{r}
=\sum_{\eta \in \bar{\Omega}_{(g, n, \beta)}} \frac{\mathbf{m}(\eta)}{|E q(\eta)|} \Phi_{\eta_{*}} \Delta^{!}\left(\left[\overline{\mathcal{M}}\left(\tilde{X}, D, \Gamma_{1}\right)\right]^{\mathrm{vir}} \times\left[\overline{\mathcal{M}}\left(\mathbb{P}\left(N_{Z / X} \oplus \mathcal{O}_{Z}\right), D, \Gamma_{2}\right)\right]^{\mathrm{vir}}\right), \\
\text { where } \beta \in H_{2}(X) .
\end{array}
$$

The set $\bar{\Omega}_{(g, n, \beta)}$ is an equivalence set $\Omega_{(g, n, \beta)} / \sim_{\text {equ }}$. The set $\Omega_{(g, n, \beta)}$ is a collection of admissible triples $\eta=\left(\Gamma_{1}, \Gamma_{2}, I\right)$ satisfying:

(1) $\Gamma_{1}$ and $\Gamma_{2}$ are admissible weighted graphs for $(X, D)$ and $\left(\mathbb{P}\left(N_{Z / X} \oplus \mathcal{O}_{Z}\right), D\right)$ respectively.

(2) $\Gamma_{1}$ and $\Gamma_{2}$ are required to have identical number of roots, say $r$ roots. The weight of $i$-th root in $\Gamma_{1}$ and $\Gamma_{2}$ must be identical, for $i=1, \cdots, r$.

(3) If one glues all corresponding roots of $\Gamma_{1}$ and $\Gamma_{2}$, then the new graph must be connected. 
(4) $n=\# \operatorname{legs}\left(\Gamma_{1}\right)+\# \operatorname{legs}\left(\Gamma_{2}\right)$.

(5) $I$ is a rule concerning the ordering of the union of legs in $\Gamma_{1}$ and $\Gamma_{2}$.

(6) (Genus constraint) $g(\eta):=g\left(\Gamma_{1}\right)+g\left(\Gamma_{2}\right)+r-1$ must equal $g$.

(7) (Homology constraint) $\pi_{*}\left(b\left(\Gamma_{1}\right)\right)+p_{2 *}\left(b\left(\Gamma_{2}\right)\right)=\beta$ and some other restrictions; see Section 3 in [25].

Given a permutation $\sigma \in S_{r}, \eta^{\sigma}$ is defined by reordering $r$ roots. Define $\eta_{1} \sim \eta_{2}$ if and only if $\eta_{1}^{\sigma}=\eta_{2}$ for some $\sigma \cdot \bar{\Omega}_{(g, n, \beta)}$ is the equivalence class of this relation. Define

$\mathrm{Eq}(\eta):=\#\left\{\sigma \in S_{r} \mid \eta^{\sigma}=\eta\right\}$ and $\mathbf{m}(\eta):=$ the product of the weights of the roots in $\Gamma_{1}$.
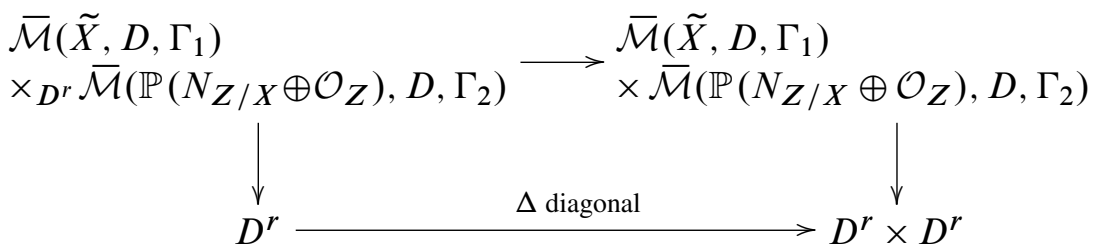

$\Phi_{\eta}: \overline{\mathcal{M}}\left(\tilde{X}, D, \Gamma_{1}\right) \times_{D^{r}} \overline{\mathcal{M}}\left(\mathbb{P}\left(N_{Z / X} \oplus \mathcal{O}_{Z}\right), D, \Gamma_{2}\right) \rightarrow \overline{\mathcal{M}}\left(\mathcal{W}_{0}, g, n, \beta\right)$ is to glue two relative stable morphisms.

One can also apply the deformation to the normal cone to $D \subset \tilde{X}$ :

$$
\tilde{W}:=\mathrm{Bl}_{D \times\{0\}} \tilde{X} \times \mathbb{C} \rightarrow \mathbb{C} .
$$

Note $\tilde{W}_{t} \cong \tilde{X}$ for $t \neq 0$ and

$$
\tilde{W}_{0}=\tilde{X} \underset{\mathbb{P}\left(N_{Z / X}\right) \frac{\bigcup}{\mathbb{P}\left(N_{Z / X} \oplus \mathcal{O}_{Z}\right)}}{\bigcup}
$$

where $\overline{\mathbb{P}\left(N_{Z / X} \oplus \mathcal{O}_{Z}\right)}$ is the blow up of $\mathbb{P}\left(N_{Z / X} \oplus \mathcal{O}_{Z}\right)$ along $Z$. This space can also be viewed as a $\mathbb{P}^{1}$-bundle over $\mathbb{P}\left(N_{Z / X}\right)$ :

$$
\tilde{p_{2}}: \overline{\mathbb{P}\left(N_{Z / X} \oplus \mathcal{O}_{Z}\right)}=\mathbb{P}_{D}\left(\mathcal{O}_{N_{Z / X}}(1) \oplus \mathcal{O}\right) \rightarrow \mathbb{P}\left(N_{Z / X}\right)=D
$$

Our goal is to compare the virtual classes $\left[\overline{\mathcal{M}}\left(\mathcal{W}_{0}, 0, n, \beta\right)\right]^{\mathrm{vir}}$ and $\left[\overline{\mathcal{M}}\left(\widetilde{\mathcal{W}}_{0}, 0, n, \pi^{!} \beta\right)\right]^{\mathrm{vir}}$. By the degeneration formula, the main issue is to realize all contributions from $\left(\mathbb{P}\left(N_{Z / X} \oplus \mathcal{O}_{Z}\right), D\right)$ and $\left(\overline{\mathbb{P}\left(N_{Z / X} \oplus \mathcal{O}_{Z}\right)}, D\right)$. 


\subsection{Relative case}

Let $\pi_{Y}: \tilde{Y} \rightarrow Y:=\mathbb{P}\left(N_{Z / X} \oplus \mathcal{O}_{Z}\right)$ be the blow up along $Z$. Given an admissible graph $\widetilde{\Gamma}$ for $(\tilde{Y}, D)$, define the admissible graph $\pi_{Y_{*}}(\widetilde{\Gamma})$ for $(Y, D)$ by the following:

(1) All information of $\pi_{Y_{*}}(\widetilde{\Gamma})$ is identical to that of $\widetilde{\Gamma}$ except the weight function $b$.

(2) We have a commutative diagram:

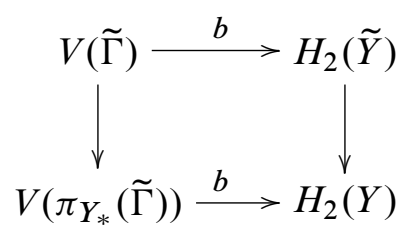

Lemma 4.3 Suppose $N_{Z / X}$ is convex, and the genus weight function of $\widetilde{\Gamma}$ is a zero function. Assume $\overline{\mathcal{M}}\left(Y, D, \pi_{Y_{*}}(\widetilde{\Gamma})\right)$ makes sense. Then $\overline{\mathcal{M}}(\tilde{Y}, D, \widetilde{\Gamma}) \rightarrow$ $\overline{\mathcal{M}}\left(Y, D, \pi_{Y_{*}}(\tilde{\Gamma})\right)$ have compatible perfect obstruction theories.

Proof Let $\mathcal{T}$ be the Artin stack parametrizing the possible target of relative stable maps to $(Y, D)$ and $(\tilde{Y}, D)$. The perfect obstruction theory on $\overline{\mathcal{M}}\left(Y, D, \pi_{Y_{*}}(\tilde{\Gamma})\right)$ is induced from a relative perfect obstruction theory on

$\overline{\mathcal{M}}\left(Y, D, \pi_{Y_{*}}(\widetilde{\Gamma})\right) \rightarrow \mathcal{T} \times \mathfrak{M}_{g(\tilde{\Gamma}), k}$, where $k=$ number of legs + number of roots.

Since $\mathcal{T} \times \mathfrak{M}_{g(\widetilde{\Gamma}), k}$ is a smooth Artin stack, we have a right exact sequence:

$$
\operatorname{RelOb}(f) \rightarrow \operatorname{Ob}(C, f) \rightarrow 0,
$$

where $\operatorname{Ob}(C, f)$ refers to the obstruction space of $\overline{\mathcal{M}}\left(Y, D, \pi_{Y_{*}}(\widetilde{\Gamma})\right)$ at the point $\left[C \stackrel{f}{\rightarrow} Y_{l} \rightarrow Y\right]$.

$$
\begin{gathered}
\phi: \overline{\mathcal{M}}(\tilde{Y}, D, \tilde{\Gamma}) \rightarrow \overline{\mathcal{M}}\left(Y, D, \pi_{Y_{*}}(\tilde{\Gamma})\right) \\
{\left[\widetilde{C} \stackrel{\tilde{f}}{\rightarrow} \tilde{Y}_{l} \rightarrow \tilde{Y}\right] \mapsto\left[C \stackrel{f}{\rightarrow} Y_{l} \rightarrow Y\right]}
\end{gathered}
$$

We have a diagram of right exact sequence:

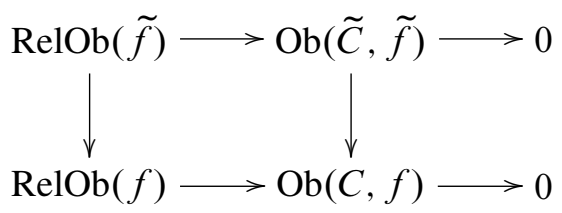

Step $1 \operatorname{RelOb}(\tilde{f}) \rightarrow \operatorname{RelOb}(f)$ is surjective. 
There is a natural diagram of exact sequences:

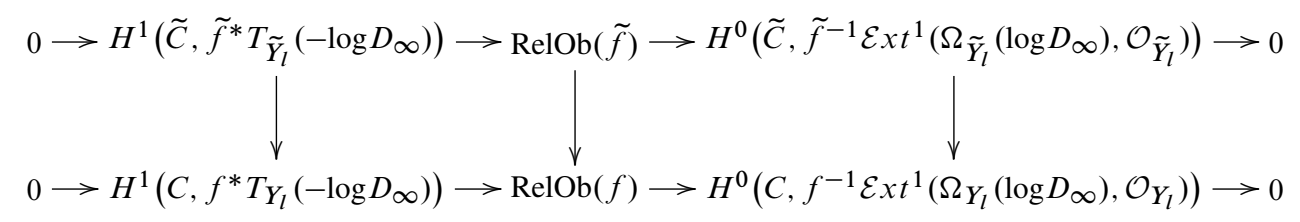

$\mathcal{E} x t^{1}\left(\Omega_{\tilde{Y}_{l}}\left(\log D_{\infty}\right), \mathcal{O}_{\tilde{Y}_{l}}\right)$ and $\mathcal{E} x t^{1}\left(\Omega_{Y_{l}}\left(\log D_{\infty}\right), \mathcal{O}_{Y_{l}}\right)$ are both supported on $\operatorname{Sing}\left(\widetilde{Y}_{l}\right)=\operatorname{Sing}\left(Y_{l}\right)=\bigcup_{i=0}^{l-1} D_{i}$, and these two sheaves are canonically isomorphic to each other. Therefore, it remains to show the first vertical arrow is surjective. We also have another exact sequence

$$
0 \rightarrow T_{\widetilde{Y}_{l}}\left(-\log D_{\infty}\right) \rightarrow T_{Y_{l}}\left(-\log D_{\infty}\right) \rightarrow Q_{N_{Z / X}} \rightarrow 0
$$

where $Q_{N_{Z / X}}$ is the universal quotient bundle on $\mathbb{P}_{Z}\left(N_{Z / X}\right)$. Now the proof proceeds as the second part of the proof in Proposition 3.2. This concludes Step 1.

Step $2 \operatorname{RelOb}(\tilde{f}) \rightarrow \operatorname{RelOb}(f)$ is surjective $\Longrightarrow \mathrm{Ob}(\tilde{C}, \tilde{f}) \rightarrow \mathrm{Ob}(C, f)$ is surjective.

By Lemma 3.5 and Proposition 3.6, there exists a relative perfect obstruction theory on $\overline{\mathcal{M}}(\tilde{Y}, D, \widetilde{\Gamma}) \rightarrow \overline{\mathcal{M}}\left(Y, D, \pi_{Y_{*}}(\widetilde{\Gamma})\right)$. Moreover, it is compatible with two existing obstruction theories on the two moduli spaces.

There is an induced map on admissible triples: $\Psi: \Omega_{(0, n, \pi ! \beta)} \rightarrow \Omega_{(0, n, \beta)}$, where $\Psi\left(\Gamma_{1}, \Gamma_{2}, I\right)=\left(\Gamma_{1}, \pi_{Y_{*}}\left(\Gamma_{2}\right), I\right)$.

Lemma 4.4 Suppose $\Psi\left(\Gamma_{1}, \Gamma_{2}, I\right)=\left(\Gamma_{1}, \Gamma_{3}, I\right)$, then we have $b\left(\Gamma_{2}\right)=\pi_{Y}^{!}\left(b\left(\Gamma_{3}\right)\right) \in$ $H_{2}(\tilde{Y})$.

Proof Since $\pi_{Y_{*}}\left(b\left(\Gamma_{2}\right)\right)=b\left(\Gamma_{3}\right)$, it is enough to prove $b\left(\Gamma_{2}\right) \bullet D_{\infty}=0$ in $\tilde{Y}$, where $l_{\infty}: D_{\infty}=\mathbb{P}\left(N_{Z / X}\right) \hookrightarrow \widetilde{Y}$ has normal bundle $\mathcal{O}_{N_{Z / X}}(-1)$. Let $l_{0}: D_{0}=$ $\mathbb{P}\left(N_{Z / X}\right) \hookrightarrow \tilde{Y}$ be the divisor which has normal bundle $\mathcal{O}_{N_{Z / X}}(1)$.

We have $b\left(\Gamma_{2}\right)=l_{0_{*}}\left(\tilde{p}_{2 *} b\left(\Gamma_{2}\right)\right)+f$, where $f$ is a multiple of the fiber class of $\tilde{p}_{2}$. It remains to show $f=0$.

$$
\widetilde{Y} \stackrel{\widetilde{p_{2}}}{\rightarrow} D=D_{0} \stackrel{\iota_{0}}{\rightarrow} \tilde{Y}
$$

$\left(\Gamma_{1}, \Gamma_{2}, I\right) \in \Omega_{(0, n, \pi ! \beta)}$ implies:

$$
\begin{gathered}
\pi^{!} \beta=b\left(\Gamma_{1}\right)+\tilde{p}_{2 *} b\left(\Gamma_{2}\right), \\
b\left(\Gamma_{1}\right) \bullet D \text { in } \tilde{X}=b\left(\Gamma_{2}\right) \bullet D_{0} \text { in } \tilde{Y} .
\end{gathered}
$$


Therefore we have

$$
\begin{aligned}
0=\pi ! \beta \bullet D & =\left(b\left(\Gamma_{1}\right) \bullet D \text { in } \tilde{X}\right)+\left(\tilde{p}_{2 *} b\left(\Gamma_{2}\right) \bullet D \text { in } \tilde{X}\right) \\
& =\left(b\left(\Gamma_{2}\right) \bullet D_{0} \text { in } \tilde{Y}\right)-\left(l_{0 *} \widetilde{p_{2 *}} b\left(\Gamma_{2}\right) \bullet D_{0} \text { in } \tilde{Y}\right) \\
& =f \bullet D_{0} \text { in } \tilde{Y} .
\end{aligned}
$$

Given $\left(\Gamma_{1}, \Gamma, I\right) \in \Omega_{(0, n, \beta)}$, define

$\Psi^{-1}(\Gamma)=\left\{\Gamma_{2}\right.$ is a admissible weighted graph for $(\tilde{Y}, D)$

$$
\left.\mid\left(\Gamma_{1}, \Gamma_{2}, I\right) \in \Omega_{(0, n, \pi ! \beta)} \text { such that } \Psi\left(\Gamma_{1}, \Gamma_{2}, I\right)=\left(\Gamma_{1}, \Gamma, I\right)\right\} .
$$

It is straightforward to check that $\Psi^{-1}(\Gamma)$ depends on $(0, n, \beta)$, but is independent of $\Gamma_{1}$ and $I$. Given $\widetilde{\Gamma} \in \Psi^{-1}(\Gamma)$, we have a natural map $\overline{\mathcal{M}}(\widetilde{Y}, D, \widetilde{\Gamma}) \rightarrow \overline{\mathcal{M}}(Y, D, \Gamma)$. Note that $\operatorname{vdim}_{\mathbb{C}} \overline{\mathcal{M}}(\tilde{Y}, D, \widetilde{\Gamma})=\operatorname{vdim}_{\mathbb{C}} \overline{\mathcal{M}}(Y, D, \Gamma)$, and the weight functions $g$ of $\widetilde{\Gamma}$ and $\Gamma$ are both zero functions.

On the other hand, there is a canonical preimage $\pi_{Y}^{!}(\Gamma) \in \Psi^{-1}(\Gamma)$, which is characterized by:

(1) All information of $\pi_{Y}^{!}(\Gamma)$ is identical to that of $\Gamma$ except the weight function $b$.

(2) We have a commutative diagram:

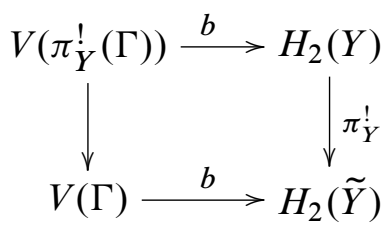

Lemma 4.5 If $Z \subset X$ is of type $I$, then we have:

(1) $\phi_{*}\left[\overline{\mathcal{M}}\left(\tilde{Y}, D, \pi_{Y}^{!}(\Gamma)\right)\right]^{\mathrm{vir}}=[\overline{\mathcal{M}}(Y, D, \Gamma)]^{\mathrm{vir}}$.

(2) $\phi_{*}[\overline{\mathcal{M}}(\tilde{Y}, D, \tilde{\Gamma})]^{\mathrm{vir}}=0$ when $\pi_{Y}^{!}(\Gamma) \neq \widetilde{\Gamma} \in \Psi^{-1}(\Gamma)$.

Proof For the first statement, the submanifold $Z$ will be moved so that the technical assumption in Proposition 3.15 is satisfied:

supp(each irreducible component of $\left.\mathbb{C}_{\mathbf{N}}\right) \cap U$ is nonempty in $\mathbf{N}$,

where $\mathbf{N}=\overline{\mathcal{M}}(Y, D, \Gamma)$ and $U$ is a collection of relative stable maps supported away from the submanifold $Z$.

For each irreducible component of $\mathbb{C}_{\mathbf{N}}$, we pick a point in the support of the cone

$$
C_{i} \stackrel{f_{i}}{\rightarrow} Y_{l_{i}} \rightarrow Y, \text { for } i=1, \cdots, k
$$


Since the subbundle $\mathcal{F}$ is generated by global sections, we have $\bigoplus_{S} \mathcal{O}_{Z} \rightarrow \mathcal{F} \rightarrow 0$.

$$
\mathbb{C}^{s} \stackrel{p \text { projection }}{\longleftarrow} Z \times \mathbb{C}^{s}=\operatorname{Vect}\left(\bigoplus_{s} \mathcal{O}_{Z}\right) \stackrel{\theta \text { smooth }}{\longrightarrow} \operatorname{Vect}(\mathcal{F}) \stackrel{\text { closed }}{\hookrightarrow} \operatorname{Vect}\left(N_{Z / X}\right) \stackrel{\text { open }}{\hookrightarrow} Y \text {. }
$$

Because $p$ is proper, $p\left(\theta^{-1}\left(\operatorname{Vect}(\mathcal{F}) \cap f_{i}\left(C_{i}\right)\right)\right)$ is closed with dimension $\leq 1+s-$ $\operatorname{rk}(F) \leq s-1$. There exists a point $q \in \mathbb{C}^{s}$ such that $q \notin p\left(\theta^{-1}\left(\operatorname{Vect}(\mathcal{F}) \cap f_{i}\left(C_{i}\right)\right)\right)$, for all $i$.

The point $q$ induces a section of $N_{Z / X} \rightarrow Z$, say $q(Z) \subset \operatorname{Vect}\left(N_{Z / X}\right)$. We have $q(Z) \cap f_{i}\left(C_{i}\right)=\varnothing$. Move the submanifold $Z$ to $q(Z)$, and notice that the technical assumption is satisfied for the case $\mathrm{Bl}_{q(Z)} Y \rightarrow Y$. By Proposition 3.15, we obtain $\phi_{*}\left[\overline{\mathcal{M}}\left(\tilde{Y}, D, \pi_{Y}^{!}(\Gamma)\right)\right]^{\mathrm{vir}}=[\overline{\mathcal{M}}(Y, D, \Gamma)]^{\mathrm{vir}}$.

For the second statement, the argument is the same, but one applies Corollary 3.16 instead.

Proposition 4.6 Suppose $Z \subset X$ is of type $I$. Then $\phi_{*}\left[\overline{\mathcal{M}}\left(\tilde{\mathcal{W}}_{0}, 0, n, \pi^{!} \beta\right)\right]^{\mathrm{vir}}=$ $\left[\overline{\mathcal{M}}\left(\mathcal{W}_{0}, 0, n, \beta\right)\right]^{\mathrm{vir}}$.

Proof By Lemma 4.5 and Degeneration formula from blow-up, it remains to check

$$
\begin{aligned}
\mathrm{Eq}\left(\Gamma_{1}, \pi_{Y}^{!}(\Gamma), I\right) & =\mathrm{Eq}\left(\Gamma_{1}, \Gamma, I\right) \\
\mathbf{m}\left(\Gamma_{1}, \pi_{Y}^{!}(\Gamma), I\right) & =\mathbf{m}\left(\Gamma_{1}, \Gamma, I\right)
\end{aligned} \quad \forall\left(\Gamma_{1}, \Gamma, I\right) \in \bar{\Omega}_{(0, n, \beta)},
$$

which is straightforward.

Proposition 4.7 Suppose $Z \subset X$ is of type II. Then $\phi_{*}\left[\overline{\mathcal{M}}\left(\tilde{\mathcal{W}}_{0}, 0, n, \pi^{!} \beta\right)\right]^{\mathrm{vir}}=$ $\left[\overline{\mathcal{M}}\left(\mathcal{W}_{0}, 0, n, \beta\right)\right]^{\mathrm{vir}}$.

Proof Due to the property of $Z$, any vector bundle over $Z$ is automatically convex. It suffices to prove Lemma 4.5 for type II. First one observes that there is a natural fibration

$$
\overline{\mathcal{M}}(Y, D, \Gamma) \rightarrow Z \text { with nonsingular fibers } \cong \overline{\mathcal{M}}\left(\mathbb{P}^{m}, \mathbb{P}^{m-1}, \Gamma\right),
$$

where $m=\operatorname{rk}\left(N_{Z / X}\right)$. In particular, $\overline{\mathcal{M}}(Y, D, \Gamma)$ is a smooth DM-stack. Therefore the technical assumption of Proposition 3.15 is equivalent to saying :

any point in $\overline{\mathcal{M}}(Y, D, \Gamma)$ can be moved so that the corresponding curve is supported away from $Z$. 
The point will be moved along the fiber $\overline{\mathcal{M}}\left(\mathbb{P}^{m}, \mathbb{P}^{m-1}, \Gamma\right)$, so we may assume $Z=$ point, $Y=\mathbb{P}^{m}$.

Given a point in the moduli space

$$
C \stackrel{f}{\rightarrow} Y_{l} \rightarrow Y=\mathbb{P}^{m}=\mathbb{P}^{m-1} \cup \mathbb{C}^{m},
$$

pick a point $q=\left(v_{1}, v_{2}, \cdots, v_{m}\right) \in \mathbb{C}^{m}$ such that $q \notin f(C)$.

It is easy to find an one parameter family $v: \mathbb{C} \rightarrow P G L\left(\mathbb{C}^{m+1}\right)=\operatorname{Aut}\left(\mathbb{P}^{m}\right)$ so that:

(1) When $t=1, v(1) \circ(C, f)$ is supported away from the origin $Z$.

(2) $v(t)$ preserves the divisor $\mathbb{P}^{m-1} \subset \mathbb{P}^{m}$.

(3) $v(t)$ doesn't change the contact order of $(C, f)$ with the divisor.

Proposition 4.6 and Proposition 4.7 immediately imply Theorem 1.5 and Theorem 1.4.

Proof of Theorem 1.4 Since the degeneration used here comes from the deformation to the normal cone from blow-up construction, all insertions involved in the equality, ie cohomology classes from $X$ and the vector bundle $V$, can be lifted to the degeneration.

\subsection{Descendant invariants}

The upshot of this subsection is the following:

Corollary 4.8 Suppose each connected component of the submanifold $Z=\bigsqcup_{i} Z_{i} \subset$ $X$ is of type I or type II. If $a_{i} \leq \max \left(2, \operatorname{codim}_{\mathbb{C}}(Z, X)-1\right)$ for all $i$, then we have

$$
\left\langle\tau_{a_{1}} \alpha_{1}, \cdots, \tau_{a_{n}} \alpha_{n}\right\rangle_{0, n, \beta}^{X}=\left\langle\tau_{a_{1}} \pi^{*} \alpha_{1}, \cdots, \tau_{a_{n}} \pi^{*} \alpha_{n}\right\rangle_{0, n, \pi ! \beta}^{\tilde{X}} \text {, where } \alpha_{i} \in H^{*}(X) .
$$

If there are too many cotangent line classes $\psi_{i}$, the previous equality of descendant invariants is not expected to hold. This is because the stabilization of the domain curve via $\varphi: \overline{\mathcal{M}}_{0, n}\left(\tilde{X}, \pi^{!} \beta\right) \rightarrow \overline{\mathcal{M}}_{0, n}(X, \beta)$ causes $\psi_{i} \neq \varphi^{*} \psi_{i}$. Indeed, $\psi_{i}-\varphi^{*} \psi_{i}$ corresponds to boundary strata in the moduli space.

Given $\tilde{\beta} \in H_{2}(\tilde{X})$, if $\overline{\mathcal{M}}_{0, n}\left(X, \pi_{*} \tilde{\beta}\right)$ makes sense, then define

$$
\begin{aligned}
\left\langle\tau_{a_{1}} \tau_{b_{1}}^{\prime} \gamma_{1}, \cdots,\right. & \left.\tau_{a_{n}} \tau_{b_{n}}^{\prime} \gamma_{n}\right\rangle_{0, n, \tilde{\beta}}^{\tilde{X}} \\
& :=\int_{\left[\overline{\mathcal{M}}_{0, n}(\tilde{X}, \tilde{\beta})\right]^{\mathrm{vir}}} \psi_{1}^{a_{1}} \cap \varphi^{*} \psi_{1}^{b_{1}} \cap \cdots \cap \psi_{n}^{a_{n}} \cap \varphi^{*} \psi_{n}^{b_{n}} \cap \mathrm{ev}^{*}\left(\bigotimes_{i=1}^{n} \gamma_{i}\right),
\end{aligned}
$$


where $\varphi: \overline{\mathcal{M}}_{0, n}(\tilde{X}, \tilde{\beta}) \rightarrow \overline{\mathcal{M}}_{0, n}\left(X, \pi_{*} \tilde{\beta}\right)$ and $\gamma_{i} \in H^{*}(\tilde{X})$.

Theorem 1.5 implies

$$
\left\langle\tau_{b_{1}}^{\prime} \pi^{*} \alpha_{1}, \cdots, \tau_{b_{n}}^{\prime} \pi^{*} \alpha_{n}\right\rangle_{0, n, \pi ! \beta}^{\tilde{X}}=\left\langle\tau_{b_{1}} \alpha_{1}, \cdots, \tau_{b_{n}} \alpha_{n}\right\rangle_{0, n, \beta}^{X} .
$$

However, $\psi_{i} \neq \varphi^{*} \psi_{i}$. In order to prove Corollary 4.8 , we will show that the correction term vanishes if there are not too many cotangent line classes.

We may assume $Z$ is connected. One can blow up successively to deduce results for disconnected submanifold $Z$. Given an arbitrary map $\pi: Y \rightarrow X$, suppose $\pi_{*}(\beta)=$ $0 \in H_{2}(X)$, where $\beta \in H_{2}(Y)$. Therefore we have a diagram:

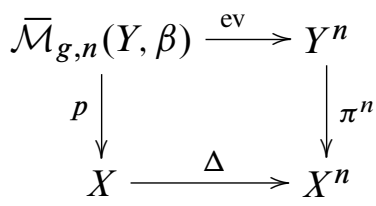

Suppose $\quad \Theta \in H^{*}\left(\overline{\mathcal{M}}_{g, n}(Y, \beta)\right), \quad \alpha_{i} \in H^{*}(X)$ and $\quad \gamma_{i} \in H^{*}(Y)$.

For convenience, denote $\overline{\mathcal{M}}_{g, n}(Y, \beta)$ by $\mathbf{M}$.

Lemma 4.9 We have:

$$
\left.\int_{[\mathbf{M}]^{\mathrm{vir}}} \Theta \cap \mathrm{ev}^{*}\left(\bigotimes_{i}\left(\gamma_{i} \cap \pi^{*} \alpha_{i}\right)\right)=\int_{p_{*}\left([\mathbf{M}]^{\mathrm{vir}} \cap \Theta \cap \mathrm{ev}^{*}\right.}\left(\bigotimes_{i} \gamma_{i}\right)\right) \cap_{i} \alpha_{i}
$$

Proof This follows from the projection formula.

We will set $Y$ as $\tilde{X}$, and $\beta$ as $d e$, where $e$ is the $\mathbb{P}^{1}$ line class in the exceptional divisor and $d$ is an integer. The previous lemma says we can freely reorganize factors from $H^{*}(X)$.

Lemma 4.10 If $d>0$, then $\left\langle\tau_{k} \pi^{*} \alpha, \gamma\right\rangle_{0,2, d e}^{\tilde{X}}=0$ when $k \leq \max \left(1, \operatorname{codim}_{\mathbb{C}}(Z, X)-2\right)$.

Proof By the previous lemma,

$$
\left\langle\tau_{k} \pi^{*} \alpha, \gamma\right\rangle_{0,2, d e}^{\tilde{X}}=\left\langle\tau_{k}, \pi^{*} \alpha \cap \gamma\right\rangle_{0,2, d e}^{\tilde{X}} .
$$

The case $k=0$ is trivial. When $k=1$,

$$
\left\langle\tau_{1}, \pi^{*} \alpha \cap \gamma\right\rangle_{0,2, d e}^{\tilde{X}}=(2 g-2+2)\left\langle\pi^{*} \alpha \cap \gamma\right\rangle_{0,1, d e}^{\tilde{X}}=0 .
$$

If the invariant doesn't vanish, then we have $\operatorname{deg}\left(\pi^{*} \alpha \cap \gamma\right) \leq \operatorname{dim} X-1$. Otherwise, the pull back of $\pi^{*} \alpha \cap \gamma$ to the exceptional divisor $D$ is zero. Since $\overline{\mathcal{M}}_{0,2}(\tilde{X}, d e) \cong$ 
$\overline{\mathcal{M}}_{0,2}(D, d e)$, the invariant vanishes. On the other hand, $k+\operatorname{deg}\left(\pi^{*} \alpha \cap \gamma\right)=$ $\operatorname{vdim}_{\mathbb{C}} \overline{\mathcal{M}}_{0,2}(\tilde{X}, d e)=\operatorname{dim} X-3+2+d\left(\operatorname{codim}_{\mathbb{C}}(Z, X)-1\right)$. Therefore,

$$
k \geq d\left(\operatorname{codim}_{\mathbb{C}}(Z, X)-1\right) \geq \operatorname{codim}_{\mathbb{C}}(Z, X)-1 .
$$

Given $\varphi: \overline{\mathcal{M}}_{0, n}(\tilde{X}, \widetilde{\beta}) \rightarrow \overline{\mathcal{M}}_{0, n}\left(X, \pi_{*} \tilde{\beta}\right)$, the boundary strata associated to $\psi_{1}-\varphi^{*} \psi_{1}$ are given by the clutching morphism from:

$$
\overline{\mathcal{M}}_{0,1+\{q\}}(\tilde{X}, d e) \text { and } \overline{\mathcal{M}}_{0,\left\{q^{\prime}\right\}+(n-1)}(\tilde{X}, \tilde{\beta}-d e),
$$

where $d$ runs through all positive integers. And then glue two points $q$ and $q^{\prime}$,

$$
\overline{\mathcal{M}}_{0,1+\{q\}}(\tilde{X}, d e) \times_{\tilde{X}} \overline{\mathcal{M}}_{0,\left\{q^{\prime}\right\}+(n-1)}(\tilde{X}, \widetilde{\beta}-d e) \rightarrow \overline{\mathcal{M}}_{0, n}(\tilde{X}, \widetilde{\beta}) .
$$

Now we pull back line bundles $\mathbb{L}_{1}$ and $\varphi^{*} \mathbb{L}_{1}$ on $\overline{\mathcal{M}}_{0, n}(\tilde{X}, \tilde{\beta})$ to $\overline{\mathcal{M}}_{0,1+\{q\}}(\tilde{X}, d e) \times \tilde{X}$ $\overline{\mathcal{M}}_{0,\left\{q^{\prime}\right\}+(n-1)}(\tilde{X}, \widetilde{\beta}-d e)$. We have:

(1) $\left.\left(\mathbb{L}_{1}\right.$ on $\left.\overline{\mathcal{M}}_{0, n}(\tilde{X}, \tilde{\beta})\right)\right|_{\text {strata }}=\mathbb{L}_{1}$ on $\overline{\mathcal{M}}_{0,1+\{q\}}(\tilde{X}$, de $)$.

(2) $\left.\left(\varphi^{*} \mathbb{L}_{1}\right.$ on $\left.\overline{\mathcal{M}}_{0, n}(\tilde{X}, \tilde{\beta})\right)\right|_{\text {strata }}=\theta^{*} \mathbb{L}_{q^{\prime}}$ on $\overline{\mathcal{M}}_{0,\left\{q^{\prime}\right\}+(n-1)}(\tilde{X}, \tilde{\beta}-d e)$, where $\theta: \overline{\mathcal{M}}_{0,\left\{q^{\prime}\right\}+(n-1)}(\tilde{X}, \widetilde{\beta}-d e) \rightarrow \overline{\mathcal{M}}_{0,\left\{q^{\prime}\right\}+(n-1)}\left(X, \pi_{*} \widetilde{\beta}\right)$.

Suppose $[\Delta]^{\vee}=\sum_{i} T_{i} \otimes T_{i}^{\vee}$ is the Kunneth decomposition of the Poincare dual of the class $[\Delta]$, where $\Delta: \tilde{X} \rightarrow \tilde{X} \times \tilde{X}$ is the diagonal map.

Lemma 4.11 Suppose $\pi: \tilde{X}=\tilde{X} \rightarrow X$ is an arbitrary blow-up. If $1 \leq a_{1} \leq$ $\max \left(2, \operatorname{codim}_{\mathbb{C}}(Z, X)-1\right)$, then we have

$$
\begin{aligned}
\left\langle\tau_{a_{1}} \tau_{b_{1}}^{\prime} \pi^{*} \alpha_{1}, \tau_{a_{2}} \tau_{b_{2}}^{\prime} \gamma_{2}, \cdots, \tau_{a_{n}} \tau_{b_{n}}^{\prime} \gamma_{n}\right\rangle_{0, n, \tilde{\beta}}^{\tilde{X}} & \\
& =\left\langle\tau_{a_{1}+b_{1}} \pi^{*} \alpha_{1}, \tau_{a_{2}} \tau_{b_{2}}^{\prime} \gamma_{2}, \cdots, \tau_{a_{n}} \tau_{b_{n}}^{\prime} \gamma_{n}\right\rangle_{0, n, \tilde{\beta}}^{\tilde{X}},
\end{aligned}
$$

where $\alpha_{1} \in H^{*}(X)$ and $\gamma_{i} \in H^{*}(\tilde{X})$.

Proof Use induction on $a_{1}$. The analysis of $\psi_{1}-\varphi^{*} \psi_{1}$ shows:

$$
\begin{aligned}
\left\langle\tau_{a_{1}} \tau_{b_{1}}^{\prime}\right. & \left.\pi^{*} \alpha_{1}, \tau_{a_{2}} \tau_{b_{2}}^{\prime} \gamma_{2}, \cdots, \tau_{a_{n}} \tau_{b_{n}}^{\prime} \gamma_{n}\right\rangle_{0, n, \tilde{\beta}}^{\tilde{X}} \\
& =\left\langle\tau_{a_{1}-1} \tau_{b_{1}+1}^{\prime} \pi^{*} \alpha_{1}, \tau_{a_{2}} \tau_{b_{2}}^{\prime} \gamma_{2}, \cdots, \tau_{a_{n}} \tau_{b_{n}}^{\prime} \gamma_{n}\right\rangle_{0, n, \tilde{\beta}}^{\tilde{X}} \\
& +\sum_{d>0} \sum_{T_{i}}\left\langle\tau_{a_{1}-1} \pi^{*} \alpha_{1}, T_{i}\right\rangle_{0,2, d e}^{\tilde{X}} \bullet\left\langle\tau_{b_{1}}^{\prime} T_{i}^{\vee}, \tau_{a_{2}} \tau_{b_{2}}^{\prime} \gamma_{2}, \cdots, \tau_{a_{n}} \tau_{b_{n}}^{\prime} \gamma_{n}\right\rangle_{0, n, \tilde{\beta}-d e}^{\tilde{X}} .
\end{aligned}
$$

As $a_{1}-1 \leq \max \left(1, \operatorname{codim}_{\mathbb{C}}(Z, X)-2\right)$, by Lemma $4.10\left\langle\tau_{a_{1}-1} \pi^{*} \alpha_{1}, T_{i}\right\rangle_{0,2, d e}^{\tilde{X}}=0$. 
Proof of Corollary 4.8 In the previous lemma, set $\gamma_{i}=\pi^{*} \alpha_{i}, \widetilde{\beta}=\pi^{!} \beta$ and $b_{1}=$ $b_{2}=\cdots=b_{n}=0$. Then apply the lemma to $a_{1}, a_{2}, \cdots, a_{n}$, this shows

$$
\left\langle\tau_{a_{1}} \pi^{*} \alpha_{1}, \cdots, \tau_{a_{n}} \pi^{*} \alpha_{n}\right\rangle_{0, n, \pi ! \beta}^{\tilde{X}}=\left\langle\tau_{a_{1}}^{\prime} \pi^{*} \alpha_{1}, \cdots, \tau_{a_{n}}^{\prime} \pi^{*} \alpha_{n}\right\rangle_{0, n, \pi ! \beta}^{\tilde{X}} .
$$

Now it follows from Theorem 1.5.

\subsection{Examples and remarks}

Example 4.12 Given any projective manifold $X$, here we provide several ways to find a submanifold $Z \subset X$, so that $N_{Z / X}$ is generated by global sections.

(1) Embed $X$ in a homogeneous space $\mathcal{P}$, and pick an arbitrary submanifold $Y \subset \mathcal{P}$. By Bertini's Theorem, one can perturb $Y$ so that $Y$ are transversal to $X$. Then take $Z=X \cap Y$.

(2) Take any vector bundle $V$ over $X$ and an ample line bundle $L$. When $n>>0$, $V \otimes L^{\otimes n}$ is generated by global sections. Take a regular section $s \in H^{0}(X, V \otimes$ $\left.L^{\otimes n}\right)$, and let $Z=s^{-1}(0)$.

(3) Suppose $L_{1}, L_{2}, \cdots, L_{m}$ are line bundles over $X$, and are generated by global sections. Take a regular section $s \in H^{0}\left(X, \bigoplus_{i=1}^{m} L_{i}\right)$, and let $Z=s^{-1}(0)$. Then $Z$ is a complete intersection of $X$.

Example 4.13 Suppose $L_{1}, L_{2}, \cdots, L_{m}$ are line bundles over $Z$, and each $L_{i}$ is generated by global sections. Let $X=\mathbb{P}_{Z}\left(\bigoplus_{i=1}^{m} L_{i} \oplus \mathcal{O}_{Z}\right)$ be the projective completion, and $\widetilde{X}$ be the blow-up along $Z$. Let $\left(\mathbb{C}^{*}\right)^{m}$ act on $X$ and $\widetilde{X}$ fiberwise. In principle, one can use virtual localization to express all GW-invariants of $\tilde{X}$ and $X$ in terms of those of $Z$, and use the calculation to prove Theorem 1.4 in this case. However, this is a formidable combinatorial task. When $Z$ is a point and $\beta \in H_{2}(X)$ has degree 2, Theorem 1.4 corresponds to Lagrangian interpolation in the localization computation after cancelling numerous terms.

Remark 4.14 Suppose $N_{Z / X}$ is generated by global sections, and $\pi: \tilde{X} \rightarrow X$ is the blow-up. Let $V$ be a convex line bundle over $X$, and $s \in H^{0}(X, V)$ is a section so that $Y:=s^{-1}(0)$ is a submanifold of $X$. It is well-known that genus-0 GW-invariants of $Y$ can be expressed by twisted invariants of $X$. If $Y$ and $Z$ is transversal in $X$, then $\pi^{*}(s) \in H^{0}\left(\tilde{X}, \pi^{*} V\right)$ is a regular section. And we have a Cartesian diagram:

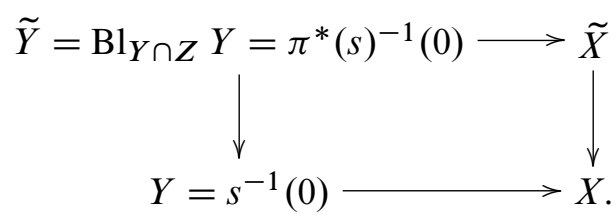


Since $\pi^{*} V$ is also a convex line bundle of $\tilde{X}$, by Theorem 1.4, we have $\{\mathrm{GW}(\tilde{Y})$ with insertions from $Y\}=\{$ twisted- $\mathrm{GW}(\tilde{X})$ with insertions from $X\}$

$$
=\{\text { twisted }-\mathrm{GW}(X)\}=\{G W(Y)\} .
$$

On the other hand, $N_{(Y \cap Z) / Y}$ is the pull back of $N_{Z / X}$, and is generated by global sections as well. This also implies $\{\mathrm{GW}(\tilde{Y})$ with insertions from $Y\}=\{\mathrm{GW}(Y)\}$.

For arbitrary blow-ups, the correction terms between GW-invariants of $\tilde{X}$ and $X$ are required. If the universal blow-up formula exists, the correction terms should also have this functoriality.

Remark 4.15 We speculate that Theorem 1.4 holds as long as $N_{Z / X}$ is convex without any additional assumption. Here we briefly discuss the technical difficulty encountered in our approach. For simplicity, assume $X=\mathbb{P}_{Z}(N \oplus \mathcal{O})$. Given any point $(C, f) \in \overline{\mathcal{M}}_{0, n}(X, \beta)$, we have $C \stackrel{f}{\rightarrow} X \stackrel{p}{\rightarrow} Z$. Because $N$ is convex, $f^{*} p^{*} N$ is generated by global sections. Therefore one can find a section of $f^{*} p^{*} N$ to move the curve so that the new curve is supported away from $Z$ in $X$.

On the other hand, suppose $\mathbb{C}_{i}$ is an irreducible component of the virtual normal cone, and $(C, f) \in \operatorname{supp}\left(\mathbb{C}_{i}\right)$. To check the technical assumption of Proposition 3.15, one has to make sure that the new curve still stays in $\operatorname{supp}\left(\mathbb{C}_{i}\right)$. A priori, $\mathbb{C}_{i}$ can be supported in a very small part of $\overline{\mathcal{M}}_{0, n}(X, \beta)$ (even if one assumes the reduced structure of $\overline{\mathcal{M}}_{0, n}(X, \beta)$ is smooth). More precisely, $\operatorname{dim} \operatorname{supp}\left(\mathbb{C}_{i}\right) \geq \operatorname{vdim}_{\mathbb{C}} \overline{\mathcal{M}}_{0, n}(X, \beta)$, and the equality can be achieved. Local analysis of Kuranish map is required if one attempts to prove in this way.

Example 4.16 In this example we will see that even if $Z \subset X$ has freedom to move to avoid any finite collection of holomorphic curves in $X$, the induced GW-invariants of $\tilde{X}$ can be different from the corresponding GW-invariants of $X$.

Consider two vector bundles on $Z=\mathbb{P}^{r}: V_{1}=\bigoplus_{i=1}^{s} \mathcal{O}_{Z}$ and $V_{2}=\bigoplus_{i=1}^{t} \mathcal{O}_{Z}(-k)$, where $s, t \geq 2$ and $k>0$. Let $X$ be the projective completion $\mathbb{P}_{Z}\left(V_{1} \oplus V_{2} \oplus \mathcal{O}_{\infty}\right)$, and $Z \subset X$ with normal bundle $V_{1} \oplus V_{2}$. Since $s \geq 2, Z$ can be moved to avoid any finite collections of holomorphic curves. Define

$$
\begin{aligned}
& \pi: \tilde{X} \rightarrow X \text { is the blow-up along } Z, \\
& Y:=\mathbb{P}_{Z}\left(V_{1} \oplus \mathcal{O}_{\infty}\right) \subset X, \\
& \pi_{Y}: \tilde{Y} \rightarrow Y \text { is the blow-up along } Z \subset Y .
\end{aligned}
$$


We have a diagram (not Cartesian):

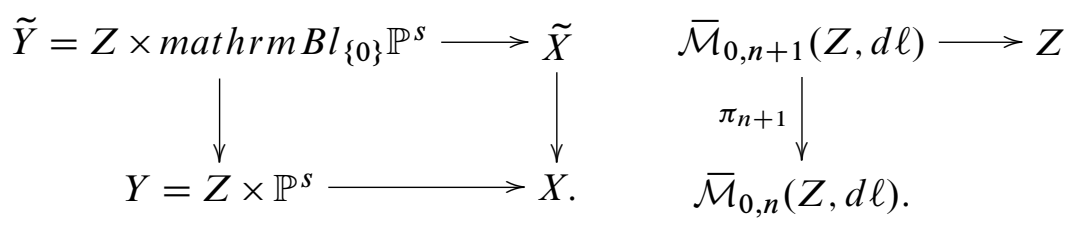

Let $[\ell]$ be the line class in $Z$. Define an obstruction bundle on $\overline{\mathcal{M}}_{0, n}(Z, d \ell)$ by $\mathcal{U}_{d}:=R^{1} \pi_{n+1_{*}} \mathrm{ev}^{*} \mathcal{O}_{Z}(-k)$.

Regard $\ell$ as a curve class in $X$ via $Z \subset X$. Let $\Phi: \overline{\mathcal{M}}_{0, n}\left(\tilde{X}, \pi^{!}[d \ell]\right) \rightarrow \overline{\mathcal{M}}_{0, n}(X,[d \ell])$ with $d>0$.

\section{Lemma 4.17}

(1) $\overline{\mathcal{M}}_{0, n}(X, d \ell) \cong \overline{\mathcal{M}}_{0, n}(Z, d \ell) \times \mathbb{P}^{s}$,

(2) $\overline{\mathcal{M}}_{0, n}\left(\tilde{X}, \pi^{!} d \ell\right) \cong \overline{\mathcal{M}}_{0, n}(Z, d \ell) \times \mathrm{Bl}_{\{0\}} \mathbb{P}^{s}$,

(3) $\mathrm{Ob}\left(\overline{\mathcal{M}}_{0, n}(X, d \ell)\right) \cong \mathcal{U}_{d} \otimes\left(\bigoplus_{t} \mathcal{O}_{\mathbb{P}}(1)\right)$,

(4) $\operatorname{Ob}\left(\overline{\mathcal{M}}_{0, n}\left(\tilde{X}, \pi^{!} d \ell\right)\right) \cong \mathcal{U}_{d} \otimes\left[\bigoplus_{t}\left(\varphi^{*} \mathcal{O}_{\mathbb{P}}(1) \otimes[-D]\right)\right]$, where $\varphi: \mathrm{B}_{\{0\}} \mathbb{P}^{s} \rightarrow$ $\mathbb{P}^{s}$ and $D$ is the exceptional divisor of $\varphi$.

Note $\Phi$ is a birational map in this case, but the natural map between obstruction bundles is not surjective. Assume the lemma, then the difference of (pushdown) virtual classes $\Phi_{*}\left[\overline{\mathcal{M}}_{0, n}\left(\tilde{X}, \pi^{!} d \ell\right)\right]^{\mathrm{vir}}-\left[\overline{\mathcal{M}}_{0, n}(X, d \ell)\right]^{\mathrm{vir}}$ in general doesn't vanish, and has nonzero contribution to GW-invariants. For example, take $s=t=k=2$ and $d=1$, then $\mathcal{U}_{d}$ is a trivial line bundle. Let $H$ be the hyperplane class of $\mathbb{P}^{2}$. Then

$$
\begin{aligned}
& \varphi_{*}\left[(H-D)^{2}\right]-H^{2}=-[\mathrm{pt}] \in A_{0}\left(\mathbb{P}^{2}\right) \\
\Longrightarrow & \Phi_{*}\left[\overline{\mathcal{M}}_{0, n}\left(\tilde{X}, \pi^{!} \ell\right)\right]^{\mathrm{vir}}-\left[\overline{\mathcal{M}}_{0, n}(X, \ell)\right]^{\mathrm{vir}} \\
& =-\left[\overline{\mathcal{M}}_{0, n}(Z, \ell)\right]^{\mathrm{vir}} \times\{\mathrm{pt}\} \in A_{*}\left(\overline{\mathcal{M}}_{0, n}(Z, \ell) \times \mathbb{P}^{2}\right),
\end{aligned}
$$

which apparently has nonzero contribution to GW-invariants. For general $s, t, k, d$, the difference of (pushdown) virtual classes is given by [ twisted $\left.-\overline{\mathcal{M}}_{0, n}(Z, d \ell)\right]^{\mathrm{vir}} \times\{\mathrm{pt}\}$, where the virtual class is twisted by the vector bundle $V_{2} \rightarrow Z$, and the characteristic class is a combination of various Chern classes.

Now we sketch the proof of Lemma 4.17. First note the normal bundle $N_{Y / X} \cong$ $\mathcal{O}_{Z}(-k) \otimes\left(\bigoplus_{t} \mathcal{O}_{\mathbb{P}^{s}}(1)\right)$. This vector bundle is $[d \ell]$-concave (but is not concave for any curve class), thus $\overline{\mathcal{M}}_{0, n}(Y, d \ell)$ is a path-connected component of $\overline{\mathcal{M}}_{0, n}(X, d \ell)$. Let $\left[\ell_{1}\right]=[\ell]$ and $\left[\ell_{2}\right]$ be the line classes of $Z$ and $\mathbb{P}^{s}$. The equality $\overline{\mathcal{M}}_{0, n}(X, d \ell)=$ $\overline{\mathcal{M}}_{0, n}(Y, d \ell)$ follows from the following lemma. 
Lemma 4.18 For any $f ; \mathbb{P}^{1} \rightarrow X$, if $f\left(\mathbb{P}^{1}\right) \nsubseteq Y Y$, then

$$
f_{*}\left[\mathbb{P}^{1}\right]=a\left[\ell_{1}\right]+b\left[\ell_{2}\right] \in A_{1}(X) \cong A_{1}(Y)=A_{1}(Z) \oplus A_{1}\left(\mathbb{P}^{s}\right) \text { with } a \geq 0, b>0 \text {. }
$$

The obstruction sheaf on $\overline{\mathcal{M}}_{0, n}(Y, d \ell)$ is deduced directly from the normal bundle $N_{Y / X}$.

Lemma 4.19 Given three manifolds $Z \subset Y \subset X$, we have a diagram (not Cartesian):

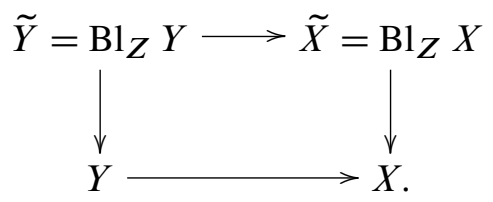

Then $N_{\tilde{Y} / \tilde{X}} \cong \pi^{*}\left(N_{Y / X}\right) \otimes[-D]$, where $D$ is the exceptional divisor of $\tilde{Y} \rightarrow Y$.

In our case, the lemma says $N_{\tilde{Y} / \tilde{X}} \cong \mathcal{O}_{Z}(-k) \otimes\left[\bigoplus_{t}\left(\varphi^{*} \mathcal{O}_{\mathbb{P}^{s}}(1) \otimes[-D]\right)\right]$, which is also a $[d \ell]-$ concave bundle. An analogue of Lemma 4.18 shows that $\overline{\mathcal{M}}_{0, n}(\tilde{Y}, d \ell)$ is the only component of $\overline{\mathcal{M}}_{0, n}\left(\tilde{X}, \pi^{!} d \ell\right)$. The analysis of obstruction sheaf is straightforward.

\section{Vanishing theorems for blow-ups}

Suppose we have a map $f: X \rightarrow Y$ between two compact complex manifolds. It is obvious that

$$
\int_{X} \alpha \wedge f^{*} \beta=0, \alpha \in H^{*}(X), \beta \in H^{*}(Y), \text { when } \operatorname{deg}_{\mathbb{R}} \beta>2 \operatorname{dim}_{\mathbb{C}} Y .
$$

However, the virtual version in general is not true (even if $X$ and $Y$ are smooth):

$$
\int_{[X]^{\mathrm{vir}}} \alpha \wedge f^{*} \beta \stackrel{?}{=} 0, \alpha \in H^{*}(X), \beta \in H^{*}(Y), \text { when } \operatorname{deg}_{\mathbb{R}} \beta>2 \operatorname{vdim}_{\mathbb{C}} Y .
$$

To rectify this situation, one has to impose the assumption that $f: X \rightarrow Y$ have compatible perfect obstruction theories. With such assumption, the vanishing result holds in the virtual version. This simple phenomenon is the starting point of vanishing theorems for blow-up in this paper.

In our convention, the empty set $\varnothing$ has dimension $=-\infty$, and $\operatorname{codim}_{\mathbb{C}}(\varnothing, S)=+\infty$ if $S$ is not empty. 
Lemma 5.1 Suppose $\mathbf{M}$ and $\mathbf{N}$ are two proper DM-stacks. Assume $\varphi: \mathbf{M} \rightarrow \mathbf{N}$ have compatible perfect obstruction theories. Let $\alpha \in A^{*}(\mathbf{M}), \beta \in A^{*}(\mathbf{N})$. Denote the virtual normal cone of $\mathbf{N}$ by $\mathbb{C}_{\mathbf{N}}$. If there exists an open substack $U \subset \mathbf{N}$ such that:

(1) $\varphi(\mathbf{M}) \cap U=\varnothing$

(2) $\operatorname{dim}\left(\mathbb{C}_{\mathbf{N}}-\left.\mathbb{C}_{\mathbf{N}}\right|_{U}\right) \leq \operatorname{dim} \mathbb{C}_{\mathbf{N}}-k$

Then $\int_{[\mathbf{M}]^{\mathrm{vir}}} \alpha \cap \varphi^{*} \beta=0$ when $\operatorname{deg} \beta>\operatorname{vdim}_{\mathbb{C}} \mathbf{N}-k$.

Proof We will adapt notation used in Section 3. Let $\mathcal{L}_{\bullet}, \mathcal{E}_{\bullet}$ and $\mathcal{F}_{\bullet}$ be the compatible perfect obstruction theories on $\mathbf{M} / \mathbf{N}, \mathbf{M}$ and $\mathbf{N}$ respectively. Recall the diagram

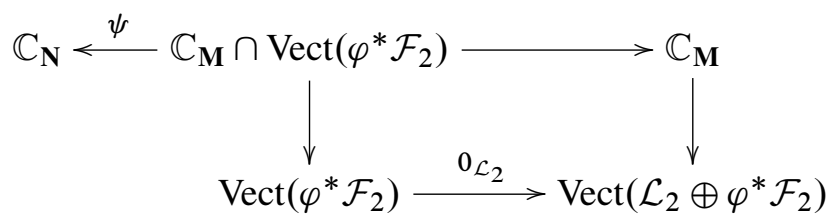

where $\mathbb{C}_{\mathbf{M}}$ and $\mathbb{C}_{\mathbf{N}}$ are virtual normal cones used to construct virtual classes. Note $\psi$ is a proper map. By abuse of notation, $\alpha$ (and $\beta$ ) will be also viewed as an element in $A^{*}\left(\mathbb{C}_{\mathbf{M}}\right)$ (and $A^{*}\left(\mathbb{C}_{\mathbf{N}}\right)$ ).

$$
\begin{aligned}
\varphi_{*}\left([\mathbf{M}]^{\mathrm{vir}} \cap \alpha \cap \varphi^{*} \beta\right)= & \varphi_{*} \circ 0_{\left(\varphi^{*} \mathcal{F}_{2}\right)}^{!} \circ 0_{\mathcal{L}_{2}}^{!}\left[\mathbb{C}_{\mathbf{M}} \cap \alpha \cap \psi^{*} \beta\right] \\
= & 0_{\mathcal{F}_{2}}^{\mathcal{F}_{2}} \circ \psi_{*} \circ 0_{\mathcal{L}_{2}}^{!}\left[\mathbb{C}_{\mathbf{M}} \cap \alpha \cap \psi^{*} \beta\right] \\
= & 0_{\mathcal{F}_{2}}^{!}\left(\beta \cap\left(\psi_{*} \circ 0_{\mathcal{L}_{2}}^{!}\left[\mathbb{C}_{\mathbf{M}} \cap \alpha\right]\right)\right), \\
& \quad \text { where } \psi_{*} \circ 0_{\mathcal{L}_{2}}^{!}\left[\mathbb{C}_{\mathbf{M}} \cap \alpha\right] \in A_{*}\left(\psi\left(\mathbb{C}_{\mathbf{M}} \cap \operatorname{Vect}\left(\varphi^{*} \mathcal{F}_{2}\right)\right)\right) .
\end{aligned}
$$

Since $\varphi(\mathbf{M}) \cap U=\varnothing$, we have $\psi\left(\mathbb{C}_{\mathbf{M}} \cap \operatorname{Vect}\left(\varphi^{*} \mathcal{F}_{2}\right)\right) \subset \mathbb{C}_{\mathbf{N}}-\left.\mathbb{C}_{\mathbf{N}}\right|_{U}$. Recall that $\operatorname{dim} \mathbb{C}_{\mathbf{N}}=\operatorname{vdim}_{\mathbb{C}}(\mathbf{N})+\operatorname{rk}\left(\mathcal{F}_{2}\right)$. Therefore $\operatorname{dim} \psi\left(\mathbb{C}_{\mathbf{M}} \cap \operatorname{Vect}\left(\varphi^{*} \mathcal{F}_{2}\right)\right) \leq \operatorname{vdim} \mathbb{C}(\mathbf{N})+$ $\operatorname{rk}\left(\mathcal{F}_{2}\right)-k$. Because $\operatorname{deg} \beta+\operatorname{rk}\left(\mathcal{F}_{2}\right)>\operatorname{vdim}_{\mathbb{C}} \mathbf{N}+\operatorname{rk}\left(\mathcal{F}_{2}\right)-k$, we know that the map $0_{\mathcal{F}_{2}}^{!} \circ(\beta \cap *): A_{\bullet}\left(\psi\left(\mathbb{C}_{\mathbf{M}} \cap \operatorname{Vect}\left(\varphi^{*} \mathcal{F}_{2}\right)\right)\right) \rightarrow A_{\bullet-\operatorname{deg} \beta-\mathrm{rk}\left(\mathcal{F}_{2}\right)}(\mathbf{N})$ must be a zero map.

There is a topological statement of Lemma 5.1. All $A_{*}(\bullet)$ in the proof must be replaced by Borel-Moore homology $H_{*}^{\mathrm{BM}}(\bullet)$. The proof is the same and is omitted.

Lemma 5.2 Suppose $\mathbf{M}$ and $\mathbf{N}$ are two proper DM-stacks. Assume $\varphi: \mathbf{M} \rightarrow \mathbf{N}$ have compatible perfect obstruction theories. Let $\alpha \in H^{*}(\mathbf{M}), \beta \in H^{*}(\mathbf{N})$. If there exists an open substack $U \subset \mathbf{N}$ such that:

(1) $\varphi(\mathbf{M}) \cap U=\varnothing$ 
(2) $\operatorname{dim}\left(\mathbb{C}_{\mathbf{N}}-\left.\mathbb{C}_{\mathbf{N}}\right|_{U}\right) \leq \operatorname{dim} \mathbb{C}_{\mathbf{N}}-k$.

Then $\int_{[\mathbf{M}]^{\mathrm{vir}}} \alpha \cap \varphi^{*} \beta=0$ when $\operatorname{deg}_{\mathbb{R}} \beta>2 \operatorname{vdim}_{\mathbb{C}} \mathbf{N}-2 k$.

\section{Remark 5.3}

(1) The second assumption $\operatorname{dim}\left(\mathbb{C}_{\mathbf{N}}-\left.\mathbb{C}_{\mathbf{N}}\right|_{U}\right) \leq \operatorname{dim} \mathbb{C}_{\mathbf{N}}-k$ only depends on $U$ and the singularities of $\mathbf{N}$, but is independent of the perfect obstruction theory $\mathcal{F}^{\bullet}$.

(2) Taking $U$ as an empty set and $k=0$, this is the vanishing result mentioned at the beginning of this section.

Corollary 5.4 Suppose $\mathbf{M}$ and $\mathbf{N}$ are two proper DM-stacks. Assume $\varphi: \mathbf{M} \rightarrow \mathbf{N}$ has compatible perfect obstruction theories. Suppose $A \subset B$ is a pair of compact complex manifolds, with a fiber diagram:

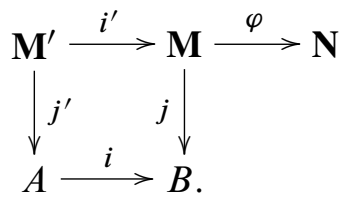

If there exists an open substack $U \subset \mathbf{N}$ such that:

(1) $\varphi \circ i^{\prime}\left(\mathbf{M}^{\prime}\right) \cap U=\varnothing$

(2) $\operatorname{dim}\left(\mathbb{C}_{\mathbf{N}}-\left.\mathbb{C}_{\mathbf{N}}\right|_{U}\right) \leq \operatorname{dim} \mathbb{C}_{\mathbf{N}}-k$.

Then $\int_{[\mathbf{M}]^{\mathrm{vir}}} j^{*}\left(\mathrm{PD}_{B} \circ i_{*}(w)\right) \cap \alpha \cap \varphi^{*} \beta=0$ when $\operatorname{deg}_{\mathbb{R}} \beta>2 \operatorname{vdim}_{\mathbb{C}} \mathbf{N}-2 k$. Here $\alpha \in H^{*}(\mathbf{M}), \beta \in H^{*}(\mathbf{N}), w \in H_{*}(A)$ and $\mathrm{PD}_{B}$ is the Poincare dual in $B$.

Proof Form a fiber diagram:

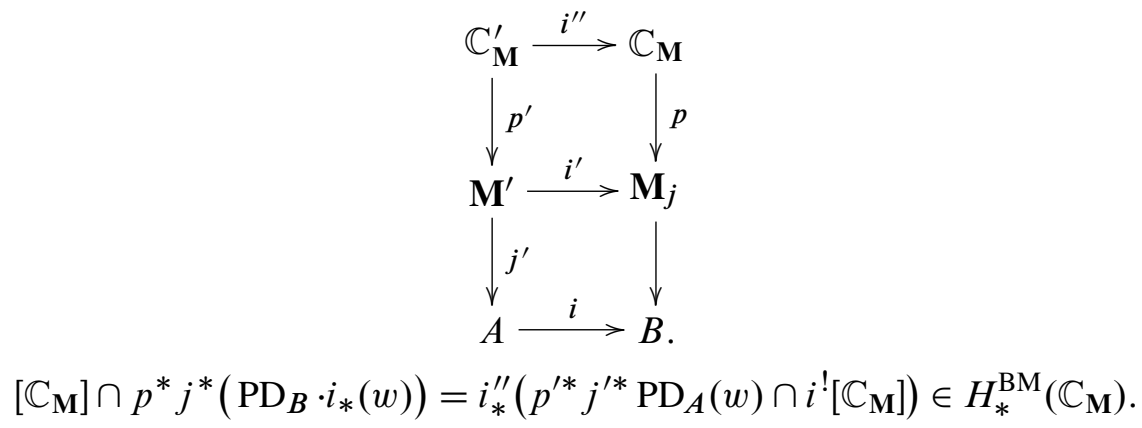


Here $i^{!}$means cap with $j^{*}\left(u_{A, B}\right)$, where $u_{A, B} \in H^{*}(B, B-A)$ is the canonical orientation class of $A \subset B$. Note $i^{!}\left[\mathbb{C}_{\mathbf{M}}\right] \in H_{*}^{\mathrm{BM}}\left(\mathbb{C}_{\mathbf{M}}^{\prime}\right)$. Let

$$
\gamma:=p^{\prime *} j^{\prime *} \operatorname{PD}_{A}(w) \cap i !\left[\mathbb{C}_{\mathbf{M}}\right] \text { and } \mathbb{C}_{\mathbf{M}}^{\prime} \cap \operatorname{Vect}\left(i^{\prime *} \varphi^{*} \mathcal{F}_{2}\right) \stackrel{\psi^{\prime}}{\rightarrow} \mathbb{C}_{\mathbf{N}}
$$

Note $\psi^{\prime}$ is a proper map. We have

$$
\int_{[\mathbf{M}]^{\mathrm{vir}}} j^{*}\left(\mathrm{PD}_{B} \circ i_{*}(w)\right) \cap \alpha \cap \varphi^{*} \beta=0_{\mathcal{F}_{2}}^{!}\left(\beta \cap\left(\psi_{*}^{\prime} \circ 0_{\mathcal{L}_{2}}^{!}[\gamma \cap \alpha]\right)\right) .
$$

Now argue as the proof of Lemma 5.1 and note the image of $\psi^{\prime}$ lies in $\mathbb{C}_{\mathbf{N}}-\left.\mathbb{C}_{\mathbf{N}}\right|_{U}$.

\subsection{Relative case}

In this subsection, we always assume $Z$ is connected. Suppose $N_{Z / X}$ is a convex bundle. We will attempt to apply the vanishing lemma to

$$
\varphi: \mathbf{M}=\overline{\mathcal{M}}_{0, n}(\tilde{X}, \tilde{\beta}) \rightarrow \overline{\mathcal{M}}_{0, n}\left(X, \pi_{*} \tilde{\beta}\right) \rightarrow \overline{\mathcal{M}}_{0, m}\left(X, \pi_{*} \tilde{\beta}\right)=\mathbf{N}, \widetilde{\beta} \neq \pi^{!} \pi_{*} \tilde{\beta},
$$

where the second arrow forgets the last $n-m$ marked points. Note

$$
\widetilde{\beta} \neq \pi^{!} \pi_{*} \widetilde{\beta} \Longleftrightarrow \widetilde{\beta}=\pi^{!} \beta+d e, d \neq 0,
$$

where $e$ is the line class in the exceptional divisor. The open substack $U \subset \mathbf{N}$ will be a collection of stable maps supported away from the submanifold $Z \subset X$. To show the composition map $\varphi$ induces compatible perfect obstruction theories, note the first map induces compatible perfect obstruction theories (Proposition 3.6), and so does the forgetful map.

Unfortunately, it is difficult to directly check the second assumption in Lemma 5.2 if $k>0$. The degeneration formula will be used to simplify the situation.

First we consider the simplest case: $Z=$ the origin $\subset X=\mathbb{P}^{r}$, with the divisor $D=\mathbb{P}^{r-1} \subset X$. Let $\mathbf{N}=\overline{\mathcal{M}}\left(\mathbb{P}^{r}, \mathbb{P}^{r-1}, \Gamma\right)$, where $\Gamma$ is an admissible graph. In this case, $\mathbf{N}$ is a smooth DM-stack.

Lemma 5.5 We have $\operatorname{codim}_{\mathbb{C}}(\mathbf{N}-U, \mathbf{N}) \geq r-1$.

Proof Define $v: \mathbb{C}^{r} \rightarrow \operatorname{PGL}\left(\mathbb{C}^{r+1}\right)=\operatorname{Aut}\left(\mathbb{P}^{m}\right)$ by:

$$
v\left(v_{1}, v_{2}, \cdots, v_{r}\right)=\left(\begin{array}{cccccc}
1 & 0 & 0 & \cdots & 0 & -v_{1} \\
0 & 1 & 0 & \cdots & 0 & -v_{2} \\
0 & 0 & 1 & \cdots & 0 & -v_{3} \\
\vdots & \vdots & \vdots & \ddots & \vdots & \vdots \\
0 & 0 & 0 & \cdots & 1 & -v_{r} \\
0 & 0 & 0 & \cdots & 0 & 1
\end{array}\right)
$$


This matrix preserves the divisor $\mathbb{P}^{r-1}$ and doesn't not change the contact order of the curve to $\mathbb{P}^{r-1}$, and therefore induces an action on $\mathbf{N}=\overline{\mathcal{M}}\left(\mathbb{P}^{r}, \mathbb{P}^{r-1}, \Gamma\right)$.

Equip $\mathbf{N}-U$ with reduced structure, denote it by $B$. Suppose $\operatorname{codim}_{\mathbb{C}}(B, \mathbf{N})<r-1$, then there exists a point

$$
\left[C \stackrel{f}{\rightarrow} Y_{l} \rightarrow Y\right] \in B
$$

so that $B$ is smooth at the point and $\operatorname{codim}_{\mathbb{C}}(B, \mathbf{N})<r-1$ near the point. Define a morphism $\sigma: \mathbb{C}^{r} \rightarrow \mathbf{N}$ by the action of $\mathbb{C}^{r}$ on

$$
\left[C \stackrel{f}{\rightarrow} Y_{l} \rightarrow Y\right]
$$

We have

$$
\sigma\left(v_{1}, \cdots, v_{r}\right) \in B \Longleftrightarrow\left(v_{1}, \cdots, v_{r}\right) \in \text { the image of the curve } C \text {. }
$$

Therefore $\operatorname{dim} \sigma^{-1}(B) \leq 1$. Take the linearized map of $\sigma$ :

$$
\begin{gathered}
\left.T \sigma\right|_{Z}:\left.\left.T \mathbb{C}^{r}\right|_{Z} \rightarrow T \mathbf{N}\right|_{\left[C \stackrel{f}{\rightarrow} Y_{l} \rightarrow Y\right]} . \\
\left.\operatorname{dim} T \sigma\right|_{Z} ^{-1}\left(\left.T B\right|_{\left[C \stackrel{f}{\rightarrow} Y_{l} \rightarrow Y\right]}\right) \leq 1 .
\end{gathered}
$$

Therefore $\operatorname{codim}_{\mathbb{C}}(B, \mathbf{N}) \geq \operatorname{codim}_{\mathbb{C}}\left\{\left.T \sigma\right|_{Z} ^{-1}\left(\left.T B\right|_{\left[C \stackrel{f}{\rightarrow} Y_{l} \rightarrow Y\right]}\right),\left.T \mathbb{C}^{r}\right|_{Z}\right\} \geq r-1$, which is a contradiction.

Now we can control the codimension for type II cases.

Lemma 5.6 Suppose $Z$ is of type II. Let $Y=\mathbb{P}\left(N_{Z / X} \oplus \mathcal{O}\right), D=\mathbb{P}\left(N_{Z / X}\right)$ and $\mathbf{N}=\overline{\mathcal{M}}_{0, n}(Y, D, \Gamma)$. Then

$$
\operatorname{codim}_{\mathbb{C}}\left(\mathbb{C}_{\mathbf{N}}-\left.\mathbb{C}_{\mathbf{N}}\right|_{U}, \mathbb{C}_{\mathbf{N}}\right) \geq \operatorname{rk}\left(N_{Z / X}\right)-1 .
$$

Proof $\mathbf{N}$ is a smooth DM-stack. It suffices to show $\operatorname{codim}_{\mathbb{C}}(\mathbf{N}-U, \mathbf{N}) \geq \operatorname{rk}\left(N_{Z / X}\right)-1$. The fibration $\mathbf{N}=\overline{\mathcal{M}}_{0, n}(Y, D, \Gamma) \rightarrow Z$ is locally trivial, and therefore reduces the problem to the fiber. Now it follows from the previous lemma.

Lemma 5.7 Suppose $N_{Z / X}$ is convex and there is a subbundle $\mathcal{F} \subset N_{Z / X}$ generated by global sections. Let $Y=\mathbb{P}\left(N_{Z / X} \oplus \mathcal{O}\right), D=\mathbb{P}\left(N_{Z / X}\right)$ and $\mathbf{N}=\overline{\mathcal{M}}_{0, n}(Y, D, \Gamma)$. Then there exists a section $q \in H^{0}\left(Z, N_{Z / X}\right)$, so that

$$
\operatorname{codim}_{\mathbb{C}}\left(\mathbb{C}_{\mathbf{N}}-\left.\mathbb{C}_{\mathbf{N}}\right|_{U_{q}}, \mathbb{C}_{\mathbf{N}}\right) \geq \operatorname{rk}(\mathcal{F})-1,
$$

where $U_{q} \subset \mathbf{N}$ is a collection of relative stable maps supported away from $q(Z)$ in $Y$. 
In particular, if $N_{Z / X}$ is generated by global sections, then we have a good bound of the codimension $\geq \operatorname{rk}\left(N_{Z / X}\right)-1$. Now the goal is to prove Lemma 5.7. $(\bullet)^{\text {red }}$ means the reduced structure.

Lemma 5.8 Suppose $f: \mathcal{A} \rightarrow \mathcal{B}$ is a morphism of separated DM-stacks of finite type over $\mathbb{C}$. Then $\mathcal{A}$ can be split as finite disjoint union $\mathcal{A}=\bigsqcup_{\text {finite }} \mathcal{A}_{i}$, so that:

(1) For each $i, \mathcal{A}_{i}$ is irreducible and locally closed in $\mathcal{A}$ and then equipped with reduced structure.

(2) Set $f_{i}: \mathcal{A}_{i} \rightarrow \overline{f\left(\mathcal{A}_{i}\right)}$. Then $f_{i}^{-1}\left(f_{i}(a)\right)$ has $\operatorname{dim} \mathcal{A}_{i}-\operatorname{dim} \overline{f\left(\mathcal{A}_{i}\right)}$ for all $a \in \mathcal{A}_{i}$.

Proof Use the induction on the number of irreducible components of top dimension in the domain. Suppose $D$ is an irreducible component of top dimension in $\mathcal{A}$. The induced map $f: D^{\text {red }} \rightarrow \overline{f(D)}^{\text {red }}$ is a dominant morphism of integral DM-stack of finite type over $\mathbb{C}$. There exists an open substack $\mathcal{U} \subset D^{\text {red }}$, such that for any $y \in f\left(D^{\text {red }}\right), \operatorname{dim} \mathcal{U}_{y}=\operatorname{dim} D-\operatorname{dim} \overline{f(D)}$. It remains to consider $f: \mathcal{A}-D \rightarrow \mathcal{B}$ and $f: D^{\text {red }}-\mathcal{U} \rightarrow \mathcal{B}$.

Given an admissible graph $\Gamma$ for $(Y, D)$ and assume $\overline{\mathcal{M}}(Y, D, \Gamma)$ exists. Define

$$
\overline{\mathcal{M}}(Y, \Gamma):=\prod_{v \in V(\Gamma)} \overline{\mathcal{M}}_{g(v), \# \operatorname{legs}(v)+\# \operatorname{roots}(v)}(Y, b(v)) .
$$

Because $\Gamma$ is relatively connected, $\overline{\mathcal{M}}(Y, \Gamma)$ makes sense and is the moduli space of (disconnected)-stable maps in $Y$. Note here we have used the condition: if $|V(\Gamma)|>1$, then each vertex $v \in V(\Gamma)$ has at least one root and $b(v) \neq 0$.

There is a natural map

$$
\overline{\mathcal{M}}(Y, D, \Gamma) \rightarrow \overline{\mathcal{M}}(Y, \Gamma) .
$$

But there is no natural arrow between two obstruction theories. The universal curve of $\overline{\mathcal{M}}(Y, \Gamma)$ is

$$
\begin{aligned}
& \overline{\mathcal{M}}(Y, \Gamma)^{\mathrm{univ}} \\
& =\coprod_{v \in V(\Gamma)} \frac{\overline{\mathcal{M}}(Y, \Gamma)}{\overline{\mathcal{M}}_{g(v), \# \operatorname{legs}(v)+\# \operatorname{roots}(v)}(Y, b(v))} \times \overline{\mathcal{M}}_{g(v), \# \operatorname{legs}(v)+\# \operatorname{roots}(v)+1}(Y, b(v)) .
\end{aligned}
$$

Note the coarse moduli space of $\overline{\mathcal{M}}(Y, \Gamma)$ is projective, as shown in [6].

Lemma 5.9 Suppose $\mathcal{A}$ is a separated DM-stack of finite type over $\mathbb{C}$ with pure dimension. Assume $\mathcal{F} \subset N_{Z / X}$ is a subbundle generated by global sections. 
Given $\varphi: \mathcal{A} \rightarrow \overline{\mathcal{M}}(Y, \Gamma)$, then there always exists a section $q \in H^{0}\left(Z, N_{Z / X}\right)$, such that $\operatorname{codim}_{\mathbb{C}}\left(\mathcal{A}-\left.\mathcal{A}\right|_{\mathcal{U}_{q}}, \mathcal{A}\right) \geq \operatorname{rk}(\mathcal{F})-1$. Here $\mathcal{U}_{q} \subset \overline{\mathcal{M}}(Y, \Gamma)$ is defined by (disconnected-) stable maps supported away from $q(Z)$ in $Y$.

Proof Recall $Y=\mathbb{P}\left(N_{Z / X} \oplus \mathcal{O}\right)$ and $D=\mathbb{P}\left(N_{Z / X}\right)$. Let $r=\operatorname{rk}(\mathcal{F})$. We may assume $r \geq 2$, otherwise it is trivial.

Step 1 Use the previous lemma to split $\mathcal{A}=\bigsqcup_{\text {finite }} \mathcal{A}_{i}$. Define $\overline{\mathcal{M}}_{i}:={\overline{\varphi\left(\mathcal{A}_{i}\right)}}^{\text {red }}$, and $\bar{M}_{i}$ is the image of $\overline{\mathcal{M}}_{i}$ in the coarse moduli space $\bar{M}(Y, \Gamma)$. Assume $\bar{M}(Y, \Gamma) \hookrightarrow \mathbb{P}^{N}$ and $\operatorname{dim} \overline{\mathcal{M}}_{i}=\operatorname{dim} \bar{M}_{i}=k_{i}$.

If $k_{i} \geq r-2$, pick a subplane $\mathbb{P}^{N-k_{i}+(r-2)}$ in $\mathbb{P}^{N}$ such that $\operatorname{dim}\left(\mathbb{P}^{N-k_{i}+(r-2)} \cap \bar{M}_{i}\right)=$ $r-2$. Define two new objects by the fiber diagrams:

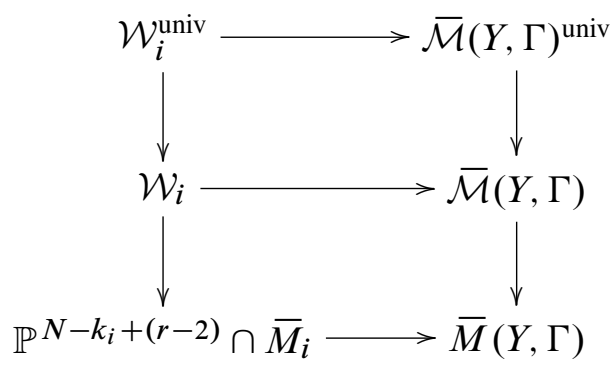

If $k_{i}<r-2$, then define $\mathcal{W}_{i}$ as $\overline{\mathcal{M}}_{i}$. Note $\operatorname{dim} \mathcal{W}_{i}^{\text {univ }} \leq r-1$ and $f_{i}: \mathcal{W}_{i}^{\text {univ }} \rightarrow Y$ is a proper map. Suppose $\bigoplus_{s} \mathcal{O}_{Z} \rightarrow \mathcal{F} \rightarrow 0$.

$$
\mathbb{C}^{s} \stackrel{p \text { projection }}{\longleftarrow} Z \times \mathbb{C}^{s}=\operatorname{Vect}\left(\bigoplus_{s} \mathcal{O}_{Z}\right) \stackrel{\theta \text { smooth }}{\longrightarrow} \operatorname{Vect}(\mathcal{F}) \stackrel{\text { closed }}{\hookrightarrow} \operatorname{Vect}\left(N_{Z / X}\right) \stackrel{\text { open }}{\hookrightarrow} Y \text {. }
$$

$$
\begin{aligned}
\operatorname{dim} p_{*} \theta^{-1}\left(f_{i}\left(\mathcal{W}_{i}^{\text {univ }}\right) \cap \operatorname{Vect}(\mathcal{F})\right) & \leq \operatorname{dim} \theta^{-1}\left(f_{i}\left(\mathcal{W}_{i}^{\text {univ }}\right) \cap \operatorname{Vect}(\mathcal{F})\right) \\
& =s-\operatorname{rk} \mathcal{F}+\operatorname{dim}\left(f_{i}\left(\mathcal{W}_{i}^{\text {univ }}\right) \cap \operatorname{Vect}(\mathcal{F})\right) \\
& \leq s-r+r-1=s-1
\end{aligned}
$$

Since there are finitely many $\mathcal{W}_{i}^{\text {univ }}$, there exists $q \in \mathbb{C}^{s}$ such that

$$
q \notin \bigcup_{i} p_{*} \theta^{-1}\left(f_{i}\left(\mathcal{W}_{i}^{\text {univ }}\right) \cap \operatorname{Vect}(\mathcal{F})\right) .
$$

Such $q$ induces an section in $H^{0}(Z, \mathcal{F}) \subset H^{0}\left(Z, N_{Z / X}\right)$.

Step 2 Claim $\operatorname{dim}\left(\overline{\mathcal{M}}_{i}-\left.\overline{\mathcal{M}}_{i}\right|_{\mathcal{U}_{q}}\right) \leq k_{i}-(r-1)$.

Suppose $k_{i} \geq r-2$. Let $\mathcal{U}_{q}^{\text {comp }}:=\overline{\mathcal{M}}(Y, \Gamma)-\mathcal{U}_{q}$ equipped with reduced structure, and $U_{q}$ be the corresponding coarse moduli. Argue by contradiction. Suppose not. 
Then $\operatorname{dim} \mathcal{U}_{q}^{\text {comp }} \cap \overline{\mathcal{M}}_{i} \geq k_{i}-(r-2)$.

$$
\begin{aligned}
& \Longrightarrow \operatorname{dim} U_{q}^{\text {comp }} \cap \bar{M}_{i} \geq k_{i}-(r-2) . \\
& \Longrightarrow \operatorname{dim} U_{q}^{\text {comp }} \cap \bar{M}_{i} \cap \mathbb{P}^{N-k_{i}+(r-2)} \neq \varnothing \text { in } \mathbb{P}^{N} . \\
& \Longrightarrow \mathcal{U}_{q}^{\text {comp }} \cap \mathcal{W}_{i} \neq \varnothing \text { in } \overline{\mathcal{M}}(Y, \Gamma) .
\end{aligned}
$$

On the other hand,

$$
q(Z) \cap f_{i}\left(\mathcal{W}_{i}^{\text {univ }}\right)=\varnothing \Longrightarrow \mathcal{W}_{i} \subset \mathcal{U}_{q}
$$

which is a contradiction.

If $k_{i}<r-2$, then a similar argument shows $\overline{\mathcal{M}}_{i} \subset \mathcal{U}_{q}$. Therefore $\overline{\mathcal{M}}_{i}-\left.\overline{\mathcal{M}}_{i}\right|_{\mathcal{U}_{q}}=\varnothing$. Step $3 \operatorname{dim}\left(\overline{\mathcal{M}}_{i}-\left.\overline{\mathcal{M}}_{i}\right|_{\mathcal{U}_{q}}\right) \leq \operatorname{dim} \overline{\mathcal{M}}_{i}-(r-1)$ and $\varphi_{i}: \mathcal{A}_{i} \rightarrow \overline{\mathcal{M}}_{i}$ has the fiber dimension $\operatorname{dim} \mathcal{A}_{i}-\operatorname{dim} \overline{\mathcal{M}}_{i}$. Therefore

$$
\begin{aligned}
\operatorname{dim}\left(\mathcal{A}_{i}-\mathcal{A}_{i} \mid \mathcal{U}_{q}\right) & \leq \operatorname{dim} \overline{\mathcal{M}}_{i}-(r-1)+\operatorname{dim} \mathcal{A}_{i}-\operatorname{dim} \overline{\mathcal{M}}_{i} \\
& =\operatorname{dim} \mathcal{A}_{i}-(r-1) \leq \operatorname{dim} \mathcal{A}-(r-1)
\end{aligned}
$$

Now it follows from $\mathcal{A}-\left.\mathcal{A}\right|_{\mathcal{U}_{q}}=\bigsqcup_{\text {finite }}\left(\mathcal{A}_{i}-\left.\mathcal{A}_{i}\right|_{\mathcal{U}_{q}}\right)$.

Proof of Lemma 5.7 Consider the composition $\mathcal{A}=\mathbb{C}_{\overline{\mathcal{M}}(Y, D, \Gamma)} \rightarrow \overline{\mathcal{M}}(Y, D, \Gamma) \rightarrow$ $\overline{\mathcal{M}}(Y, \Gamma)$. Let $\mathcal{U}_{\overline{\mathcal{M}}(Y, D, \Gamma), q}\left(\right.$ and $\mathcal{U}_{\overline{\mathcal{M}}(Y, \Gamma), q}$ ) be the (relative) stable maps supported away from $q(Z)$ in $Y$. Note $\mathcal{U}_{\overline{\mathcal{M}}(Y, D, \Gamma), q}=$ the preimage of $\mathcal{U}_{\overline{\mathcal{M}}(Y, \Gamma), q}$ under the natural map. Now it follows from the previous lemma.

The next two corollaries are the building blocks of vanishing theorems of absolute GW-invariants. According to Lemma 5.7 and Lemma 5.6, we define the codimension $\delta$ corresponding to $k$ in Lemma 5.2 as

$$
\delta= \begin{cases}\operatorname{rk}(\mathcal{F})-1 & \text { if } Z \subset \mathrm{X} \text { is of type I, } \\ \operatorname{rk}\left(N_{Z / X}\right)-1 & \text { if } Z \subset \mathrm{F} \subset \mathrm{X} \text { is of type II. }\end{cases}
$$

Recall $\pi: \tilde{Y}:=\overline{\mathbb{P}\left(N_{Z / X} \oplus \mathcal{O}_{Z}\right)} \rightarrow Y:=\mathbb{P}\left(N_{Z / X} \oplus \mathcal{O}_{Z}\right)$ is the blow up along $Z$, and $D:=\mathbb{P}\left(N_{Z / X}\right) \subset Y$. Let $A \subset[n], \alpha_{a} \in H^{*}(Y)$ for $a \in A, \gamma_{i} \in H^{*}(\tilde{Y})$ for $i \in[n]$, and $t_{*} \in H^{*}(D)$. See the paragraph before Theorem 1.7 about notation of GW-invariants.

Corollary 5.10 Suppose $Z \subset X$ is of type I or II. Let $\widetilde{\Gamma}$ be an admissible weighted graph for $(\tilde{Y}, D)$, and $\pi_{A *}$ be a composition of pushforward and the map forgetting the $[n]-A$ legs. Assume $\overline{\mathcal{M}}\left(Y, D, \pi_{A *} \tilde{\Gamma}\right)$ makes sense. 
If $\widetilde{\Gamma}$ satisfies both conditions

$$
\left\{\begin{array}{l}
\text { genus-zero weight } g: V(\widetilde{\Gamma}) \stackrel{\equiv 0}{\rightarrow} \mathbb{Z}_{\geq 0}, \\
\text { homology weight } b(v) \neq \pi^{!} \pi_{*} b(v) \text { for at least one vertex } v \in V(\widetilde{\Gamma}) .
\end{array}\right.
$$

Then we have

$$
\begin{array}{rl}
\left\langle\overrightarrow{\pi^{*} \alpha_{A}} \cdot \overrightarrow{\tau_{\left.\bullet \gamma_{[n]}\right]}} \mid t_{1}, \cdots, t_{r}\right\rangle_{\widetilde{\Gamma}}^{(\widetilde{Y}, D)}=0 & 0 \\
\text { when } \operatorname{deg} \overrightarrow{\alpha_{A}}+\sum_{i=1}^{r} \operatorname{deg} t_{i}>2 \operatorname{vdim}_{\mathbb{C}} \overline{\mathcal{M}}\left(Y, D, \pi_{A *} \widetilde{\Gamma}\right)-2 \delta .
\end{array}
$$

Proof For the type I case, let $q \in H^{0}\left(Z, N_{Z / X}\right)$ be the section found in Lemma 5.7. For the type II case, let $q$ be the zero section. Apply Lemma 5.2 to the map

$$
\varphi: \mathbf{M}=\overline{\mathcal{M}}\left(\mathrm{Bl}_{q(Z)} Y, D, \widetilde{\Gamma}\right) \rightarrow \overline{\mathcal{M}}\left(Y, D, \pi_{*} \widetilde{\Gamma}\right) \rightarrow \overline{\mathcal{M}}\left(Y, D, \pi_{A *} \widetilde{\Gamma}\right)=\mathbf{N},
$$

where $U \subset \mathbf{N}$ collects all relative stable maps supported away from $q(Z)$.

$\widetilde{\Gamma}$ has genus-zero weight $g: V(\widetilde{\Gamma}) \stackrel{\equiv}{\rightarrow} \mathbb{Z}_{\geq 0}$, and $N_{Z / X}$ is convex.

$$
\Longrightarrow \begin{aligned}
& \mathbf{M}=\overline{\mathcal{M}}\left(\mathrm{Bl}_{q(Z)} Y, D, \tilde{\Gamma}\right) \rightarrow \overline{\mathcal{M}}\left(Y, D, \pi_{*} \widetilde{\Gamma}\right) \\
& \text { have compatible perfect obstruction theories }
\end{aligned}
$$

$\overline{\mathcal{M}}\left(Y, D, \pi_{*} \widetilde{\Gamma}\right) \rightarrow \overline{\mathcal{M}}\left(Y, D, \pi_{A *} \widetilde{\Gamma}\right)=\mathbf{N}$ also induces compatible perfect obstruction theories because it forgets $[n]-A$ legs. On the other hand, $b(v) \neq \pi^{!} \pi_{*} b(v)$ for at least one $v \in V(\tilde{\Gamma})$ implies that $\varphi(\mathbf{M}) \cap U=\varnothing$. The second assumption in Lemma 5.2 follows from Lemma 5.7 and Lemma 5.6.

Corollary 5.11 Suppose $Z \subset X$ is of type I or II. Suppose $\widetilde{\Gamma}$ is an admissible weighted graph for $(\tilde{Y}, D)$ with genus-zero weight $g: V(\widetilde{\Gamma}) \stackrel{\equiv}{\rightarrow} \mathbb{Z}_{\geq 0}$. Let $\pi_{A *}$ be a composition of pushforward and the map forgetting the $[n]-A$ legs. Assume $\overline{\mathcal{M}}\left(Y, D, \pi_{A *} \tilde{\Gamma}\right)$ makes sense.

If one further assumes $j \in[n]$ and $\omega_{\{j\}} \in H^{*}(\tilde{Y})$ with $\operatorname{PD}_{\widetilde{Y}}\left(\omega_{\{j\}}\right)$ sitting inside the image of $H_{*}(E) \rightarrow H_{*}(\tilde{Y})$, where $E$ is the exceptional divisor, then we have

$$
\begin{aligned}
&\left\langle\overrightarrow{\pi^{*} \alpha_{A}} \cdot \overrightarrow{\tau_{\bullet \gamma_{[n]}}} \cdot \overrightarrow{\omega_{\{j\}}} \mid t_{1}, \cdots, t_{r}\right\rangle_{\tilde{\Gamma}}^{(\tilde{Y}, D)}=0 \\
& \text { when } \operatorname{deg} \overrightarrow{\alpha_{A}}+\sum_{i=1}^{r} \operatorname{deg} t_{i}>2 \operatorname{vdim}_{\mathbb{C}} \overline{\mathcal{M}}\left(Y, D, \pi_{A *} \tilde{\Gamma}\right)-2 \delta .
\end{aligned}
$$


Proof Apply Corollary 5.4 to:

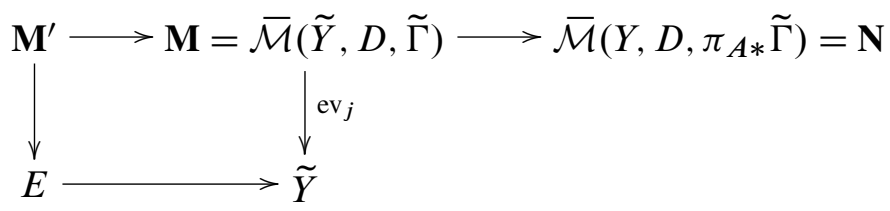

Any curve $\left[C \rightarrow \tilde{Y}_{l} \rightarrow \tilde{Y}\right] \in \mathbf{M}^{\prime}$ touches the exceptional divisor in $\tilde{Y}$, therefore the corresponding image in $\mathbf{N}$ touches $Z \subset Y$. Now it follows from Lemma 5.7 and Lemma 5.6.

\subsection{Absolute case}

Recall $\pi: \tilde{X} \rightarrow X$ is the blow up along $Z$. In the following theorem, sets $I, J, K, A$ can be empty sets. When $A$ is empty, $\operatorname{deg} \overrightarrow{\alpha_{A}}$ will be counted as zero.

Theorem 5.12 $I, J, K$ are disjoint sets with $J \subset[n]$. Suppose $Z=\left(\bigsqcup_{i \in I} Z_{i}\right) \cup$ $\left(\bigsqcup_{j \in J} Z_{j}\right) \cup\left(\bigsqcup_{k \in K} Z_{k}\right)$ is a disjoint union of submanifolds in $X$, with the following assumptions:

(1) For each $i \in I \cup J, Z_{i} \subset X$ is either of type $I$ or of type II.

(2) For each $k \in K, N_{Z_{k} / X}$ is convex.

(3) The curve class $\tilde{\beta}=\pi^{!} \beta+\sum_{i \in I} d_{i} e_{i}+\sum_{j \in J} d_{j} e_{j}+\sum_{k \in K} d_{k} e_{k}$ with $d_{i} \neq$ 0 for all $i \in I$, and $0 \neq \beta \in H_{2}(X)$. Here $e_{\bullet}$ are the line classes in the corresponding exceptional divisors.

(4) $\overrightarrow{\omega_{J}}$ is a collection of cohomology classes in $H^{*}(\tilde{X})$. And $\operatorname{PD}_{\tilde{X}}\left(\omega_{j}\right)$ lies in the image of $H_{*}\left(E_{j}\right) \rightarrow H_{*}(\tilde{X})$.

For $i \in I \cup J$, define

$$
\delta_{i}= \begin{cases}\operatorname{rk}(\mathcal{F})-1 & \text { if } Z_{i} \subset X \text { is of type } I, \\ & \text { and } \mathcal{F} \subset N_{Z_{i} / X} \text { is generated by global sections. } \\ \operatorname{rk}\left(N_{Z_{i} / X}\right)-1 & \text { if } Z_{i} \subset X \text { is of type II. }\end{cases}
$$

Then $\left\langle\overrightarrow{\pi^{*} \alpha_{A}} \cdot \overrightarrow{\tau_{\bullet} \gamma_{[n]}} \cdot \overrightarrow{\omega_{J}}\right\rangle_{0, n, \tilde{\beta}}^{\tilde{X}}=0$

$$
\text { when } \operatorname{deg} \overrightarrow{\alpha_{A}}>2 \operatorname{vdim}_{\mathbb{C}} \overline{\mathcal{M}}_{0, A}(X, \beta)-2 \sum_{i \in I} \delta_{i}-2 \sum_{j \in J} \delta_{j}
$$

Here $\overrightarrow{\alpha_{A}}$ is a collection of cohomology classes from $X$ with $A \subset[n]$, and $\overrightarrow{\tau_{\bullet} \gamma_{[n]}}$ are arbitrary descendant insertions of $\tilde{X}$. 
Proof For $i \in I \cup J \cup K$, define

$$
Y_{i}:=\mathbb{P}_{Z_{i}}\left(N_{Z_{i} / X} \oplus \mathcal{O}_{Z_{i}}\right), \quad \pi_{i}: \tilde{Y}_{i}:=\mathrm{Bl}_{Z_{i}} Y_{i} \rightarrow Y_{i}, \quad D_{i}:=\mathbb{P}_{Z_{i}}\left(N_{Z_{i} / X}\right) \subset Y_{i}
$$

Apply the degeneration for blow-up:

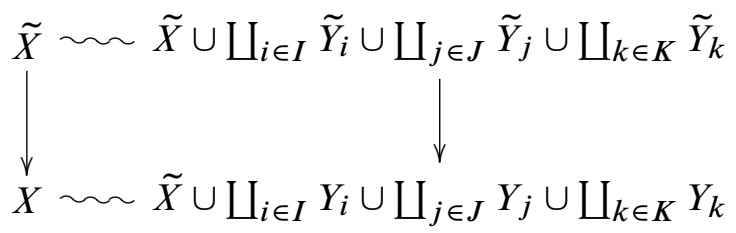

Given $\left(\widetilde{\Gamma},\left\{\widetilde{\Gamma}_{i}\right\}_{i \in I},\left\{\widetilde{\Gamma}_{j}\right\}_{j \in J},\left\{\widetilde{\Gamma}_{k}\right\}_{k \in K}\right) \in \Omega_{0, n, \tilde{\beta}}$, we have

$$
\left(\pi_{A *} \widetilde{\Gamma},\left\{\pi_{i, A, *} \widetilde{\Gamma}_{i}\right\}_{i \in I},\left\{\pi_{j, A, *} \tilde{\Gamma}_{j}\right\}_{j \in J},\left\{\pi_{k, A, *} \tilde{\Gamma}_{k}\right\}_{k \in K}\right) \in \Omega_{0, n, \beta},
$$

where $\pi_{i, A, *}, \pi_{j, A, *}$ and $\pi_{k, A, *}$ are the compositions of pushforward and the map forgetting marked legs corresponding to $[n]-A$ in the absolute case. Note for each $i \in I, \widetilde{\Gamma}_{i}$ can't be empty, and $b\left(\widetilde{\Gamma}_{i}\right)=\pi_{i}^{!} b\left(\pi_{i, A, *} \widetilde{\Gamma}_{i}\right)+d_{i} e_{i}$.

Let $\theta_{0}^{*}, \theta_{i}^{*}, \theta_{j}^{*}, \theta_{k}^{*}$ refer to the distribution of insertions to various pieces $\tilde{X}, \tilde{Y}_{i}, \tilde{Y}_{j}, \tilde{Y}_{k}$. We can choose the distribution so that:

(1) $\theta_{i}^{*} \overrightarrow{\pi^{*} \alpha_{A}}, \theta_{j}^{*} \overrightarrow{\pi^{*} \alpha_{A}}$ and $\theta_{k}^{*} \overrightarrow{\pi^{*} \alpha_{A}}$ are the pull back of cohomology classes from $Y_{i}, Y_{j}$ and $Y_{k}$.

(2) $\theta^{*} \overrightarrow{\omega_{J}}$ are distributed to the corresponding divisors in $\tilde{Y}_{j}$, for $j \in J$.

Argue by contradiction, suppose the invariant is not zero, then there exists

$$
\begin{aligned}
& \left(\widetilde{\Gamma},\left\{\widetilde{\Gamma}_{i}\right\}_{i \in I},\left\{\tilde{\Gamma}_{j}\right\}_{j \in J},\left\{\widetilde{\Gamma}_{k}\right\}_{k \in K}\right) \in \Omega_{0, n, \tilde{\beta}}, \\
& \overrightarrow{t_{i}} \in H^{*}\left(D_{i}^{\# \text { \#rots of } \tilde{\Gamma}_{i}}\right), \text { for } i \in I \cup J \cup K,
\end{aligned}
$$

so that

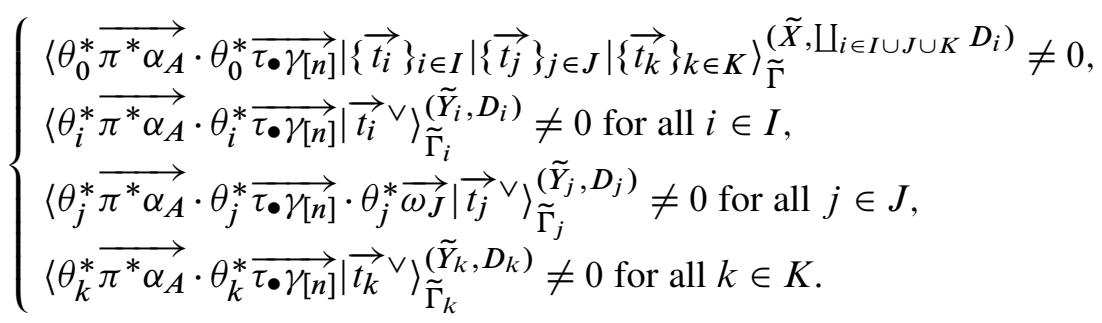


Given $i \in I \cup J \cup K$, define $\operatorname{deg} \theta_{i}^{*} \overrightarrow{\pi^{*} \alpha_{A}}:=\sum_{a \in A \cap \operatorname{legs} \text { of } \widetilde{\Gamma}_{i}} \operatorname{deg} \alpha_{a}$, then we have $\operatorname{deg}{\overrightarrow{t_{i}}}^{\vee}+\operatorname{deg} \theta_{i}^{*} \overrightarrow{\pi^{*} \alpha_{A}} \leq 2 \operatorname{vdim}_{\mathbb{C}} \overline{\mathcal{M}}\left(Y_{i}, D_{i}, \pi_{i, A, *} \widetilde{\Gamma}_{i}\right)-2 \delta_{i} \quad$ by Corollary 5.10, $\operatorname{deg}{\overrightarrow{t_{j}}}^{\vee}+\operatorname{deg} \theta_{j}^{*} \overrightarrow{\pi^{*} \alpha_{A}} \leq 2 \operatorname{vdim}_{\mathbb{C}} \overline{\mathcal{M}}\left(Y_{j}, D_{j}, \pi_{j, A, *} \widetilde{\Gamma}_{j}\right)-2 \delta_{j} \quad$ by Corollary 5.11, $\operatorname{deg}{\overrightarrow{t_{k}}}^{\vee}+\operatorname{deg} \theta_{k}^{*} \overrightarrow{\pi^{*} \alpha_{A}} \leq 2 \operatorname{vdim}_{\mathbb{C}} \overline{\mathcal{M}}\left(Y_{k}, D_{k}, \pi_{k, A, *} \widetilde{\Gamma}_{k}\right)$ by Lemma 5.2.

On the other hand, by the assumption on $\operatorname{deg} \overrightarrow{\alpha_{A}}$, we have

$$
\begin{aligned}
& \operatorname{deg} \theta_{0}^{*} \overrightarrow{\pi^{*} \alpha_{A}}+\sum_{i \in I \cup J \cup K} \operatorname{deg} \theta_{i}^{*} \overrightarrow{\pi^{*} \alpha_{A}}+\sum_{i \in I \cup J \cup K} \operatorname{deg}{\overrightarrow{t_{i}}}^{\vee}+\sum_{i \in I \cup J \cup K} \operatorname{deg}{\overrightarrow{t_{i}}} \\
= & \operatorname{deg}{\overrightarrow{\pi^{*} \alpha_{A}}}^{\longrightarrow} 2 \sum_{i \in I \cup J \cup K}\left(\operatorname{dim} D_{i}\right) \bullet\left(\text { \#roots of } \widetilde{\Gamma}_{i}\right) \\
> & 2 \operatorname{vdim}_{\mathbb{C}} \overline{\mathcal{M}}_{0, A}(X, \beta)-2 \sum_{i \in I} \delta_{i}-2 \sum_{j \in J} \delta_{j}+2 \sum_{i \in I \cup J \cup K}\left(\operatorname{dim} D_{i}\right) \bullet\left(\text { \#roots of } \widetilde{\Gamma}_{i}\right) \\
= & \sum_{i \in I \cup J \cup K} \operatorname{vdim}_{\mathbb{C}} \overline{\mathcal{M}}\left(Y_{i}, D_{i}, \pi_{i, A, *} \widetilde{\Gamma}_{i}\right) \\
& -2 \sum_{i \in I} \delta_{i}-2 \sum_{j \in J} \delta_{j}+2 \operatorname{vdim}_{\mathbb{C}} \overline{\mathcal{M}}\left(\tilde{X}, \coprod_{i \in I \cup J \cup K} D_{i}, \pi_{A *} \tilde{\Gamma}\right) .
\end{aligned}
$$

Combining all inequalities, we obtain

$$
\operatorname{deg} \theta_{0}^{*} \overrightarrow{\pi^{*} \alpha_{A}}+\sum_{i \in I \cup J \cup K} \operatorname{deg} \overrightarrow{t_{i}}>2 \operatorname{vdim}_{\mathbb{C}} \overline{\mathcal{M}}\left(\tilde{X}, \coprod_{i \in I \cup J \cup K} D_{i}, \pi_{A *} \tilde{\Gamma}\right) .
$$

However, the map $\overline{\mathcal{M}}\left(\tilde{X}, \bigsqcup_{i \in I \cup J \cup K} D_{i}, \tilde{\Gamma}\right) \rightarrow \overline{\mathcal{M}}\left(\tilde{X}, \bigsqcup_{i \in I \cup J \cup K} D_{i}, \pi_{A *} \tilde{\Gamma}\right)$ forgets $\{$ the marked legs of $\widetilde{\Gamma}\}-A$, and therefore induces compatible perfect obstruction theories. Thus

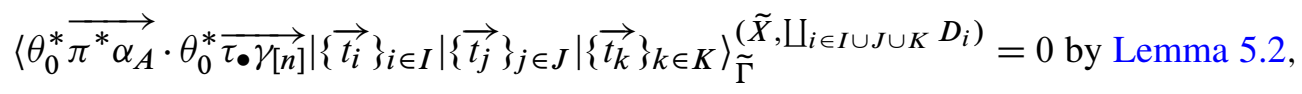

which is a contradiction.

Example 5.13 Suppose $X$ is an algebraic surface, which is neither rational nor ruled. Let $X_{0}$ be the minimal model of $X$. Since GW-invariants are deformation invariant, we may assume $\pi: X \rightarrow X_{0}$ is the blow-up at $r$ distinct points $a_{1}, \cdots, a_{r}$. Suppose $0 \neq \beta \in H_{2}\left(X_{0}\right)$.

$$
K_{X_{0}} \text { is nef } \Longrightarrow \operatorname{vdim}_{\mathbb{C}} \overline{\mathcal{M}}_{0,0}\left(X_{0}, \beta\right)=(2-3)+0-\beta \cap K_{X_{0}} \leq-1 .
$$

Assume $\tilde{\beta}=\pi^{!} \beta+\sum_{k=1}^{r} d_{k} e_{k}$, where $d_{k} \in \mathbb{Z}$. We apply the previous theorem to the case $Z=\bigsqcup_{k \in K} Z_{k}=\left\{a_{1}, \cdots, a_{r}\right\}$, with the set $I=J=A=\varnothing$. We have

$$
\operatorname{deg} \overrightarrow{\alpha_{A}}=0>-2 \geq 2 \operatorname{vdim}_{\mathbb{C}} \overline{\mathcal{M}}_{0,0}\left(X_{0}, \beta\right)
$$


By the previous theorem, $g=0$ descendant GW-invariants of $X$ are all zero if $\beta \neq 0$. Since exceptional divisors are disjoint,

$$
\begin{aligned}
& \left\langle\tau_{a_{1}} \gamma_{1}, \cdots, \tau_{a_{n}} \gamma_{n}\right\rangle_{0, n, \tilde{\beta}}^{X} \\
& \quad= \begin{cases}\text { invariants around the exceptional divisor } \mathbb{P}^{1} & \text { if } \widetilde{\beta}=d_{k} e_{k} \text { for some } k, \\
0 & \text { with } d_{k}>0 .\end{cases} \\
& \text { otherwise. }
\end{aligned}
$$

The first case can be computed by obstruction bundles.

When the arithmetic genus $p_{g}(X)>0$, this result can also be deduced from Image Localization Theorem in Lee and Parker [20] (see also Kiem and Li [16] for algebrogeometric analogue) in symplectic geometry. In fact, Image Localization Theorem is much more powerful than our argument because it can also handle higher genus $\mathrm{GW}$-invariants when $p_{g}>0$.

Example 5.14 Suppose $K_{X}$ is nef, and $Z$ is a smooth curve in $X$ with genus $g(Z) \geq 1$. Then we have zero descendant GW-invariants

$$
\langle\cdots\rangle_{0, n, \widetilde{\beta}}^{\tilde{X}} \equiv 0 \text {, when } \widetilde{\beta}=\pi^{!} \beta+d e \in H_{2}(\tilde{X}) \text { with } \beta \neq 0 \text { and } d \neq 0 \text {. }
$$

To see this, note $\operatorname{vdim}_{\mathbb{C}} \overline{\mathcal{M}}_{0,0}(X, \beta)=\operatorname{dim} X-3+0-\beta \cap K_{X} \leq \operatorname{dim} X-3$. Apply the vanishing theorem to $J=K=A=\varnothing$, then $\delta=\operatorname{codim}_{\mathbb{C}}(Z, X)-1=\operatorname{dim} X-2$. Hence $\operatorname{deg} \overrightarrow{\alpha_{A}}=0>-2 \geq 2 \operatorname{vdim}_{\mathbb{C}} \overline{\mathcal{M}}_{0,0}(X, \beta)-2 \delta$.

Example 5.15 Let $Z=\mathbb{P}^{2}$, and $X$ is the projective completion of $\mathcal{O}(-3) \oplus \mathcal{O}(-3) \rightarrow$ $Z$. This example shows Theorem 5.12 doesn't not hold for arbitrary blow-ups. Let $\pi: \tilde{X} \rightarrow X$ be the blow-up along $Z$. The exceptional divisor is $E \cong Z \times \mathbb{P}^{1}$ with normal bundle $N_{E / \tilde{X}} \cong \mathcal{O}_{Z}(-3) \otimes \mathcal{O}(-1)$. Let $\left[\ell_{1}\right]$ and $\left[\ell_{2}\right]$ be the line classes in $Z$ and $\mathbb{P}^{1}$.

$$
E \stackrel{i}{\rightarrow} \tilde{X} \stackrel{\pi}{\rightarrow} X \stackrel{p}{\rightarrow} Z
$$

Then $\pi !\left[\ell_{1}\right]=i_{*}\left(\ell_{1}-3 \ell_{2}\right)$. Consider $\overline{\mathcal{M}}_{0,1}\left(\tilde{X}, i_{*}\left(d \ell_{1}\right)\right) \rightarrow \overline{\mathcal{M}}_{0,0}\left(X, d \ell_{1}\right)$ with $d \geq 1$. Let $I=J=A=\varnothing$ in Theorem 5.12. We have $\operatorname{deg} \overrightarrow{\alpha_{A}}=0>2(1-3 d)=$ $2 \operatorname{vdim}_{\mathbb{C}} \overline{\mathcal{M}}_{0,0}\left(X, d \ell_{1}\right)$. If Theorem 5.12 holds in this example, then it implies all GW-invariants of $\overline{\mathcal{M}}_{0,1}\left(\tilde{X}, i_{*}\left(d \ell_{1}\right)\right)$ are zero.

On the other hand, $\left.\left(E+3 \pi^{*} p^{*} H_{1}\right)\right|_{E}=-H_{2} \in H^{2}(E)$, where $H_{1}$ and $H_{2}$ are hyperplane classes of $Z$ and $\mathbb{P}^{1}$ in $E$. Let $\mathcal{U}_{d} \rightarrow \overline{\mathcal{M}}_{0,0}\left(Z, d \ell_{1}\right)$ be the obstruction 
bundle associated to $\mathcal{O}(-3) \rightarrow Z$.

$$
\begin{aligned}
\left\langle 3 \pi^{*} p^{*} H_{1} \wedge\left(E+3 \pi^{*} p^{*} H_{1}\right)\right\rangle_{0,1, d \ell_{1}}^{\tilde{X}} & =-\int_{\overline{\mathcal{M}}_{0,1}\left(E, d \ell_{1}\right)^{\mathrm{vir}}} c_{\mathrm{top}}\left(\mathcal{U}_{d}\right) \cap \operatorname{ev}^{*}\left(H_{1} \otimes H_{2}\right) \\
& =-d \int_{\overline{\mathcal{M}}_{0,0}\left(Z, d \ell_{1}\right)^{\mathrm{vir}}} c_{\mathrm{top}}\left(\mathcal{U}_{d}\right)=-d \cdot K_{d} .
\end{aligned}
$$

The number $K_{d}$ has been computed by Lian, Liu and Yau [24], and is nonzero in general (eg $\left.K_{1}=3\right)$.

Example 5.16 Suppose $N_{Z / X}$ is generated by global sections and has rank $r$. Let $E$ be the exceptional divisor of $\pi: \tilde{X} \rightarrow X$. Given $a_{i} \geq 0,0 \neq \beta \in H_{2}(X)$ and $\alpha_{i} \in H^{*}(X)$, then

$$
\left\langle E^{a_{1}} \pi^{*} \alpha_{1}, E^{a_{2}} \pi^{*} \alpha_{2}, \cdots, E^{a_{n}} \pi^{*} \alpha_{n}\right\rangle_{0, n, \pi ! \beta}^{\tilde{X}}=0 \text { when } 0<\sum_{i=1}^{n} a_{i}<r-1 .
$$

To see this, we may assume $a_{1}>0$, and then apply Theorem 5.12 to:

$$
\left\{\begin{array}{l}
K=\varnothing \text { and } \delta=r-1, \\
J=\{1\} \subset[n] \text { with } \omega_{1}=E^{a_{1}}, \\
\overrightarrow{\alpha_{A}}=\left(\alpha_{1}, \cdots, \alpha_{n}\right) \text { with } A=[n] \\
\overrightarrow{\gamma_{[n]}}=\text { all remaining insertions. }
\end{array}\right.
$$

Then $\quad \operatorname{deg} \overrightarrow{\alpha_{A}}=\operatorname{vdim}_{\mathbb{C}} \overline{\mathcal{M}}_{0, n}\left(\tilde{X}, \pi^{!} \beta\right)-\sum_{i=1}^{n} a_{i}>\operatorname{vdim}_{\mathbb{C}} \overline{\mathcal{M}}_{0, n}(X, \beta)-(r-1)$

Therefore the invariant vanishes. One can use the similar argument to $\overline{\mathcal{M}}_{0, n}\left(\tilde{X}, \pi^{!} \beta\right) \rightarrow$ $\overline{\mathcal{M}}_{0, n-m}(X, \beta)$ and show that if $1 \leq m \leq n$, then

$$
\begin{aligned}
&\left\langle E^{a_{1}}, E^{a_{2}}, \cdots, E^{a_{m}}, E^{a_{m+1}} \pi^{*} \alpha_{m+1}, \cdots \cdots, E^{a_{n}} \pi^{*} \alpha_{n}\right\rangle_{0, n, \pi ! \beta}^{\tilde{X}}=0 \\
& \text { when } \sum_{i=1}^{n} a_{i}<r-1+m .
\end{aligned}
$$

If there are too many insertions coming from the exceptional divisor, then the invariant may not vanish. For example, take $X=\mathbb{P}^{3}$ and $Z=$ a point. Let $[\ell]$ be a line class in $X$. A computation in [7] shows

$$
\left\langle E^{2}, E^{2}, \cdots, E^{2}\right\rangle_{0,12,3 \ell}^{\tilde{X}}=-2332 \neq 0 .
$$




\section{Remark 5.17}

(1) Suppose $Z=Z_{1} \bigsqcup Z_{2}$ with $N_{Z_{i} / X}$ both generated by global sections. Let $\pi: \tilde{X}=\mathrm{Bl}_{Z} X \rightarrow X$ and $\pi_{i}: \tilde{X} \rightarrow X_{i}=\mathrm{Bl}_{Z_{i}} X$. To test if a GW-invariant of $\tilde{X}$ vanishes or not, using different base manifolds can yield different vanishing criteria. For example, let $\widetilde{\beta}=\pi^{!} \beta+d_{1} e_{1}+d_{2} e_{2} \in H_{2}(\tilde{X})$ with $d_{1}, d_{2}>0$. If $\alpha_{a} \in H^{*}\left(X_{1}\right)$, then

$$
\begin{aligned}
& \left\langle\overrightarrow{\left\langle\pi_{1}^{*} \alpha_{A}\right.} \cdot \overrightarrow{\left.\tau_{\bullet} \gamma_{[n]}\right]}\right\rangle_{0, n, \tilde{\beta}} \tilde{X} \\
& \quad \text { when } \operatorname{deg} \overrightarrow{\alpha_{A}}>2 \operatorname{vdim}_{\mathbb{C}} \overline{\mathcal{M}}_{0, A}\left(X_{1}, \beta+d_{1} e_{1}\right)-2 \operatorname{rk}\left(N_{Z_{2} / X}\right) .
\end{aligned}
$$

However, this result can not be deduced from the vanishing criterion for $\tilde{X} \rightarrow X$ because $\overrightarrow{\alpha_{A}}$ may not come from cohomology classes of $X$.

(2) It is not necessary to test all possible base manifolds. In Theorem 5.12, suppose $I=I_{+} \bigsqcup I_{-}$such that

$$
\widetilde{\beta}=\pi^{!} \beta+\sum_{i \in I} d_{i} e_{i}+\sum_{j \in J} d_{j} e_{j}+\sum_{k \in K} d_{k} e_{k}
$$

with $d_{i}>0$ for all $i \in I_{+}$, and $d_{i}<0$ for all $i \in I_{-}$.

A simple argument shows: if an invariant of $\tilde{X}$ satisfies the vanishing criterion for $\tilde{X} \rightarrow X$, then it automatically satisfies the vanishing criterion for $\tilde{X} \rightarrow$ $\mathrm{Bl}_{\left(Z_{I_{-}}\right)} X$, where $Z_{I_{-}}=\coprod_{i \in I_{-}} Z_{i}$.

\section{References}

[1] K Behrend, Gromov-Witten invariants in algebraic geometry, Invent. Math. 127 (1997) 601-617 MR1431140

[2] K Behrend, B Fantechi, The intrinsic normal cone, Invent. Math. 128 (1997) 45-88 MR1437495

[3] J Bryan, D Karp, The closed topological vertex via the Cremona transform, J. Algebraic Geom. 14 (2005) 529-542 MR2129009

[4] T Coates, A Givental, Quantum Riemann-Roch, Lefschetz and Serre, Ann. of Math. (2) 165 (2007) 15-53 MR2276766

[5] W Fulton, Intersection theory, Ergebnisse der Math. und ihrer Grenzgebiete [Results in Math. and Related Areas] (3) 2, Springer, Berlin (1984) MR732620

[6] W Fulton, R Pandharipande, Notes on stable maps and quantum cohomology, from: "Algebraic geometry—Santa Cruz 1995", Proc. Sympos. Pure Math. 62, Amer. Math. Soc. (1997) 45-96 MR1492534 
[7] A Gathmann, Counting rational curves with multiple points and Gromov-Witten invariants of blow-ups arXiv:AG/9609010

[8] A Gathmann, Gromov-Witten and degeneration invariants: computation and enumerative significance, $\mathrm{PhD}$ thesis, University of Hannover (1998)

[9] A Gathmann, Gromov-Witten invariants of blow-ups, J. Algebraic Geom. 10 (2001) 399-432 MR1832328

[10] T Graber, R Pandharipande, Localization of virtual classes, Invent. Math. 135 (1999) 487-518 MR1666787

[11] T Graber, R Vakil, Relative virtual localization and vanishing of tautological classes on moduli spaces of curves, Duke Math. J. 130 (2005) 1-37 MR2176546

[12] J Hu, Gromov-Witten invariants of blow-ups along points and curves, Math. Z. 233 (2000) 709-739 MR1759269

[13] J Hu, Gromov-Witten invariants of blow-ups along surfaces, Compositio Math. 125 (2001) 345-352 MR1818985

[14] J Hu, T-J Li, Y Ruan, Birational cobordism invariance of uniruled symplectic manifolds, Invent. Math. 172 (2008) 231-275 MR2390285

[15] E-N Ionel, T H Parker, The symplectic sum formula for Gromov-Witten invariants, Ann. of Math. (2) 159 (2004) 935-1025 MR2113018

[16] Y-H Kiem, J Li, Gromov-Witten invariants of varieties with holomorphic 2-forms arXiv:0707.2986

[17] B Kim, A Kresch, T Pantev, Functoriality in intersection theory and a conjecture of Cox, Katz, and Lee, J. Pure Appl. Algebra 179 (2003) 127-136 MR1958379

[18] A Kresch, Canonical rational equivalence of intersections of divisors, Invent. Math. 136 (1999) 483-496 MR1695204

[19] A Kresch, Cycle groups for Artin stacks, Invent. Math. 138 (1999) 495-536 MR1719823

[20] J Lee, T H Parker, A structure theorem for the Gromov-Witten invariants of Kähler surfaces, J. Differential Geom. 77 (2007) 483-513 MR2362322

[21] A-M Li, Y Ruan, Symplectic surgery and Gromov-Witten invariants of Calabi-Yau 3-folds, Invent. Math. 145 (2001) 151-218 MR1839289

[22] J Li, A degeneration formula of GW-invariants, J. Differential Geom. 60 (2002) 199_ 293 MR1938113

[23] J Li, G Tian, Virtual moduli cycles and Gromov-Witten invariants of algebraic varieties, J. Amer. Math. Soc. 11 (1998) 119-174 MR1467172

[24] B H Lian, K Liu, S-T Yau, Mirror principle. I, Asian J. Math. 1 (1997) 729-763 MR1621573 
[25] C-H Liu, S-T Yau, A degeneration formula of Gromov-Witten invariants with respect to a curve class for degenerations from blow-ups arXiv:math/0408147

[26] D Maulik, R Pandharipande, A topological view of Gromov-Witten theory, Topology 45 (2006) 887-918 MR2248516

[27] D McDuff, Hamiltonian $S^{1}$ manifolds are uniruled arXiv:0706.0675

[28] Y Ruan, Surgery, quantum cohomology and birational geometry, from: "Northern California Symplectic Geometry Seminar”, Amer. Math. Soc. Transl. Ser. 2 196, Amer. Math. Soc. (1999) 183-198 MR1736218

Department of Mathematics, Brandeis University

415 South Street MS 050, Waltham, MA 02454

hhlai@brandeis.edu

Proposed: Jim Bryan

Seconded: Ron Stern, Lothar Goettsche

Received: 13 March 2008

Revised: 21 July 2008 